PHYSICAL REVIEW E 94, 042602 (2016)

\title{
gु \\ Active microrheology in a colloidal glass
}

\author{
M. Gruber, ${ }^{1}$ G. C. Abade,${ }^{1}$ A. M. Puertas, ${ }^{2}$ and M. Fuchs ${ }^{1}$ \\ ${ }^{1}$ Fachbereich Physik, Universität Konstanz, 78457 Konstanz, Germany \\ ${ }^{2}$ Departamento de Física Aplicada, Universidad de Almería, 04.120 Almería, Spain
}

(Received 5 August 2016; published 11 October 2016)

\begin{abstract}
We study the dynamics of a probe particle driven by a constant force through a colloidal glass of hard spheres. This nonequilibrium and anisotropic problem is investigated using a new implementation of the mode-coupling approximation with multiple relaxation channels and Langevin dynamics simulations. A force threshold is found, below which the probe remains localized, while above it the probe acquires a finite velocity. We focus on the localized regime, comparing theory and simulations concerning the dynamics in the length scale of the cage and the properties of the transition to the delocalized regime, such as the critical power-law decay of the probe correlation function. Probe van Hove functions predicted by the theory show exponential tails reminiscent of an intermittent dynamics of the probe. This scenario is microscopically supported by simulations.
\end{abstract}

DOI: 10.1103/PhysRevE.94.042602

\section{INTRODUCTION}

In microrheology in soft-matter systems, one, or a few, colloidal probes are incorporated to the sample, and its dynamics is monitored. This was initially proposed as a substitute for conventional rheology [1] and in many cases still used in softmatter samples difficult to obtain or expensive [2,3]. However, it was soon acknowledged that a proper interpretation of microrheology requires a deep understanding of the internal dynamics of the host system, which in the original formulation is hidden in a generalization of the Stokes-Einstein relation [4]. This is particularly relevant when the probes are subject to an external force, so-called active microrheology. In this case, it can go into the nonlinear regime, where a nonaffine strain field is induced in the host system.

In a glass, the long-time (collective) dynamics is ideally arrested because the particles are caged by their own neighbors, inhibiting self-diffusion to long distances. The macroscopic system thus shows solidlike behavior, while the supercooled fluid, before the glass transition, shows (complex) viscoelastic behavior [5-7]. When a shear stress is applied to a glass, it first shows linear response when the stress is small enough, but then enters the nonlinear regime which ultimately ends in the yielding or fluidization of the system at the yield stress. The fluidized system then recovers the viscoelastic response typical of supercooled fluids [8-10].

In this paper, we aim to reproduce and rationalize the equivalent phenomenology in active microrheology. For this purpose, we analyze the motion of a probe particle pulled by a constant force through a host colloidal glass of hard spheres (HS) theoretically and by simulations. Here the caging is relevant at high densities, induced by the core-core steric hindrance [11-13]. The probe is one of the particles of the host system (selected randomly), and it is therefore also initially caged. Upon increasing the external force, it is expected to probe the elastic behavior, then enter the nonlinear regime, and, if the external force is strong enough, break free its cage, equivalent to the yielding of the glass. Previous studies on microrheology have concentrated mainly on the fluid regime. For low densities, a successful model was derived by Brady and co-workers [14-16] and confirmed by simulations, whereas at high densities, the models use mode-coupling theory to describe the bath $[17,18]$. Simulations have reached supercooled liquid states, very close to the glass transition [19], where the probe is not yet caged, reporting probe dynamics faster than diffusion, known as superdiffusion, at large forces.

In our work, the glass is described using mode-coupling theory (MCT) and for the probe dynamics we use the Smoluchowski operator. An implementation of the modecoupling approximation to forced microrheology in terms of multiple relaxation channels is developed. This is required as the original theory became unstable for forces approaching the threshold [20,21], which is solved with the formulation used here. The probe is found to be arrested for small forces, and the external force only distorts the cage, while it depins beyond a critical force. We have also run simulations with Langevin microscopic dynamics to test these results. The transition from localized to delocalized probe is found to be smoother in the simulations, and completely arrested probes are only observed for very small forces. We focus in this paper on the localized regime, comparing theory and simulations quantitatively concerning the dynamics of the probe within the cage, the distortion of the cage, and the properties of the transition to the delocalized regime.

The extended MCT used in this work is an intrinsically ensemble-averaged description of the forced probe dynamics. An alternative theoretical approach is the nonlinear Langevin equation (NLE) for the instantaneous (not ensemble-averaged) probe position [22,23], where caging enters via an effective nonequilibrium free energy and thermally activated probe dynamics can be readily incorporated. The NLE approach has been applied [23] to the study of the nonlinear response of hard-sphere colloidal suspensions to macroscopic stress. The latter is assumed to be transmitted to the particle level via a microscopic force acting on a tagged particle, the problem being reduced to the microrheological one. Therefore, their absolute yield stress may be directly related to our critical force for probe delocalization. However, the NLE formulation [23] describes only macroscopic response functions and ignores all possible anisotropies that may arise in the forced system. Here we make an effort to account for the axial symmetry induced by the external forcing in the spatial correlations of the probe particle. 
The present paper is organized as follows. Section II presents the new implementation of the mode-coupling approximation to forced microrheology. Section III contains the essentials of the Langevin dynamics simulations. Extensive comparison between theoretical and simulation results is presented and discussed in Sec. IV. We summarize our main points in Sec. V and give an outlook to future work. Technical details and a brief description of numerical solution schemes of the MCT equations are included in the appendixes.

\section{THEORETICAL DESCRIPTION}

We consider a colloidal suspension of spheres of diameter $d$. The suspension is initially in equilibrium. At $t=0$ a constant force $\mathbf{F}_{\text {ext }}=F \hat{\mathbf{z}}$ is applied to a selected particle-the probewith the same diameter as the host particles. The spheres of the host system and the probe undergo Brownian motion under the influence of thermal fluctuations in the suspending fluid. Hydrodynamic interactions between the spheres are neglected.

The motion of the probe is statistically described by its displacement distribution function $G_{s}(\mathbf{r}, t)$. It expresses the probability density for finding the probe particle at time $t$ at a distance $\mathbf{r}$ from its starting position. The average probe displacement $\delta z(t)$ along the force direction $\hat{\mathbf{z}}$, an observable of major interest, is the first moment of $G_{s}(\mathbf{r}, t)$,

$$
\delta z(t)=\langle z\rangle_{t}=\int z G_{s}(\mathbf{r}, t) d \mathbf{r}
$$

In our theoretical approach, the probe dynamics is more conveniently described in terms of the Fourier transform of $G_{s}(\mathbf{r}, t)$,

$$
\Phi_{\mathbf{q}}^{\mathrm{s}}(t)=\int e^{i \mathbf{q} \cdot \mathbf{r}} G_{S}(\mathbf{r}, t) d \mathbf{r},
$$

the so-called probe self-intermediate scattering function at the wave vector $\mathbf{q}$ or simply the probe correlator. The probe correlator $\Phi_{\mathbf{q}}^{\mathrm{s}}(t)$ provides the longitudinal displacement $\delta z(t)$ through

$$
\delta z(t)=-\left.i \frac{\partial}{\partial q} \Phi_{q \hat{\mathbf{z}}}^{\mathrm{s}}(t)\right|_{q=0},
$$

for wave vectors $\mathbf{q}=q \hat{\mathbf{z}}$ along the force direction. This can be easily verified using Eqs. (2) and (1). As implied by Eq. (3), a nonvanishing average probe displacement in force direction yields a complex-valued longitudinal probe correlator $\Phi_{q \hat{\mathbf{z}}}^{\mathrm{s}}(t)$. form

The probe correlator $\Phi_{\mathbf{q}}^{\mathrm{s}}(t)$ obeys a memory equation of the

$$
\partial_{t} \Phi_{\mathbf{q}}^{\mathrm{s}}(t)+\Gamma_{\mathbf{q}} \Phi_{\mathbf{q}}^{\mathrm{s}}(t)+\int_{0}^{t} m_{\mathbf{q}}\left(t-t^{\prime}\right) \partial_{t^{\prime}} \Phi_{\mathbf{q}}^{\mathrm{s}}\left(t^{\prime}\right) d t^{\prime}=0,
$$

with initial condition $\Phi_{\mathbf{q}}^{\mathrm{s}}(0)=1$, where $\Gamma_{\mathbf{q}}$ is the initial decay rate and the integral kernel $m_{\mathbf{q}}(t)$ accounts for memory effects arising from the interactions between the probe and the host particles. Both $\Gamma_{\mathbf{q}}$ and the memory kernel $m_{\mathbf{q}}(t)$ depend on the external force $F$ on the probe.

To be solved for the probe correlator $\Phi_{\mathbf{q}}^{\mathrm{s}}(t)$, Eq. (4) must be closed by writing the memory kernel $m_{\mathbf{q}}(t)$ as a functional of $\Phi_{\mathbf{q}}^{\mathrm{s}}(t)$,

$$
m_{\mathbf{q}}(t)=\mathscr{F}_{\mathbf{q}}\left[\boldsymbol{\Phi}^{\mathrm{s}} ; \boldsymbol{\Phi}\right] \text {. }
$$

This functional also couples the probe dynamics to the collective structural relaxation of the host system, described by the host correlator $\Phi_{q}(t)$. Here we assume $\Phi_{q}(t)$ to be isotropic and known a priori. This is a one-way coupling that neglects the influence of the driven probe particle on the host system.

In this work, the closure functional (5) will be provided by use of the mode-coupling approximation with multiple relaxation channels $[24,25]$. This approach extends previous works [18] to large forces, i.e., much stronger than those induced by thermal fluctuations in the suspending fluid. Our full microscopic model describes the probe dynamics for two control parameters: the system volume fraction $\phi$ and the external force $F$. The former controls the structural coupling in the host system, while the latter specifies the strength of the external perturbation.

\section{A. Microscopic derivation of the memory equation}

In this section we derive the memory equation (4) for the probe correlator $\Phi_{\mathbf{q}}^{\mathrm{s}}(t)$ from a microscopic description. The configuration of the $N$ hard spheres is specified by the vector $X=\left(\mathbf{r}_{1}, \ldots, \mathbf{r}_{N}\right)$, where $\mathbf{r}_{j}$ denotes the position of the $j$ th sphere. Here $\mathbf{r}_{1}$ is the position of the selected probe (labeled 1). The spheres interact via a pairwise additive potential $U(X)$. On a time scale much larger than the relaxation time of the momentum of a particle, the Brownian dynamic evolution of the configuration $X$ is assumed to be described by a probability distribution $\Psi(X, t)$ obeying the Smoluchowski equation,

$$
\partial_{t} \Psi(X, t)=\Omega \Psi(X, t),
$$

where $\Omega$ is the Smoluchowski operator.

Within the Smoluchowski description, the probe correlator $\Phi_{\mathbf{q}}^{\mathrm{s}}(t)$ may be written as

$$
\Phi_{\mathbf{q}}^{\mathrm{s}}(t)=\lim _{\infty}\left\langle\rho_{\mathbf{q}}^{\mathrm{s}} \mid e^{\Omega^{\dagger} t} \rho_{\mathbf{q}}^{\mathrm{s}}\right\rangle,
$$

where $\rho_{\mathbf{q}}^{\mathrm{s}}=\exp \left[i \mathbf{q} \cdot \mathbf{r}_{1}\right]$ is the Fourier transform of the microscopic probe density $\rho^{\mathrm{s}}(\mathbf{r})=\delta\left(\mathbf{r}-\mathbf{r}_{1}\right)$, and $\Omega^{\dagger}$ is the adjoint Smoluchowski operator,

$$
\Omega^{\dagger}=\Omega_{\mathrm{eq}}^{\dagger}+\Delta \Omega^{\dagger},
$$

composed of an equilibrium part,

$$
\Omega_{\mathrm{eq}}^{\dagger}=D_{0} \sum_{j=1}^{N}\left(\boldsymbol{\partial}_{j}+\beta \mathbf{F}_{j}\right) \cdot \boldsymbol{\partial}_{j},
$$

where $\mathbf{F}_{j}=-\boldsymbol{\partial}_{j} U(X)$, and a perturbation,

$$
\Delta \Omega^{\dagger}=\beta D_{0} \mathbf{F}_{\mathrm{ext}} \cdot \boldsymbol{\partial}_{1},
$$

due to the external force $\mathbf{F}_{\text {ext }}$ on the probe. Here $D_{0}$ is the diffusion coefficient of an isolated colloidal particle, $\beta=1 / k_{\mathrm{B}} T$, and $k_{\mathrm{B}} T$ the thermal energy. In Eq. (7) lim stands for the thermodynamic limit $N \rightarrow \infty, V \rightarrow \infty$ at constant density $n=N / V, V$ being the volume enclosing the system.

The brackets $\langle\cdot \mid \cdot\rangle$ in Eq. (7) denote the inner product,

$$
\langle f \mid g\rangle \equiv \int d X \Psi_{\mathrm{eq}}(X) f^{*}(X) g(X)
$$

of two configuration functions $f$ and $g$, weighted by the equilibrium distribution function $\Psi_{\mathrm{eq}}(X) \propto \exp [-\beta U(X)]$. The asterisk denotes complex conjugation. In our bracket notation, the bra vector $\langle\cdots$ formally represents the equilibrium distribution $\Psi_{\text {eq }}$. 
For conciseness, henceforth in the text we use dimensionless quantities. They are nondimensionalized by the corresponding characteristic scales: $d$ for length, $d^{2} / D_{0}$ for time, and $k_{\mathrm{B}} T / d$ for force.

By the Zwanzig-Mori projection operator technique [26,27] one can derive an evolution equation for $\Phi_{\mathbf{q}}^{\mathrm{s}}(t)$ of the form

$$
\partial_{t} \Phi_{\mathbf{q}}^{\mathrm{s}}(t)+\Gamma_{\mathbf{q}} \Phi_{\mathbf{q}}^{\mathrm{s}}+\int_{0}^{t} d t^{\prime} M_{\mathbf{q}}\left(t-t^{\prime}\right) \Phi_{\mathbf{q}}^{\mathrm{s}}\left(t^{\prime}\right)=0
$$

with $\Phi_{\mathbf{q}}^{\mathrm{s}}(0)=1$ and initial decay rate

$$
\Gamma_{\mathbf{q}}=-\left\langle\rho_{\mathbf{q}}^{\mathrm{s}} \mid \Omega^{\dagger} \rho_{\mathbf{q}}^{\mathrm{s}}\right\rangle=q^{2}-i \mathbf{q} \cdot \mathbf{F}_{\mathrm{ext}} \cdot
$$

The so-called mobility kernel,

$$
M_{\mathbf{q}}(t)=-\left\langle A_{\mathbf{q}}^{\mathrm{s}} \mid e^{Q_{\mathrm{s}} \Omega^{\dagger} Q_{s} t} A_{\mathbf{q}}^{\mathrm{s}}\right\rangle,
$$

is the dynamic correlation of the the stochastic force,

$$
A_{\mathbf{q}}^{\mathrm{s}}=Q_{\mathrm{s}} \Omega^{\dagger} \rho_{\mathbf{q}}^{\mathrm{s}},
$$

acting on the probe particle, where

$$
Q_{\mathrm{s}}=1-\sum_{\mathbf{q}}\left|\rho_{\mathbf{q}}^{\mathrm{s}}\right\rangle\left\langle\rho_{\mathbf{q}}^{\mathrm{s}}\right|
$$

is the projection on the subspace orthogonal to the probe density.

We then proceed by operating $\Omega^{\dagger}$ on $\rho_{\mathbf{q}}^{\mathrm{s}}$ in Eq. (15), yielding

$$
\left|A_{\mathbf{q}}^{\mathrm{s}}\right\rangle=i \mathbf{q} \cdot\left|\mathbf{H}_{\mathbf{q}}^{\mathrm{s}}\right\rangle, \quad\left\langle A_{\mathbf{q}}^{\mathrm{s}}\right|=-\left(i \mathbf{q}+\mathbf{F}_{\mathrm{ext}}\right) \cdot\left\langle\mathbf{H}_{\mathbf{q}}^{\mathrm{s}}\right|,
$$

where $\mathbf{H}_{\mathbf{q}}^{\mathrm{s}}=Q_{\mathrm{s}} \mathbf{F}_{1} \rho_{\mathbf{q}}^{\mathrm{s}}$. Note that because of its nonequilibrium component $\Delta \Omega^{\dagger}$, the backward operator $\Omega^{\dagger}$ is non-Hermitian with respect to the inner product (11) weighted by $\Psi_{\text {eq }}$ [18].

The scalar mobility kernel $M_{\mathbf{q}}(t)$ may then be written as a contraction,

$$
M_{\mathbf{q}}(t)=-\mathbf{L}_{\mathbf{q}}^{*} \cdot \mathbf{M}_{\mathbf{q}}(t) \cdot \mathbf{R}_{\mathbf{q}},
$$

of a tensor mobility kernel,

$$
\mathbf{M}_{\mathbf{q}}(t)=\left\langle\mathbf{H}_{\mathbf{q}}^{\mathrm{s}} \mid e^{Q_{s} \Omega^{\dagger} Q_{s} t} \mathbf{H}_{\mathbf{q}}^{\mathrm{s}}\right\rangle
$$

with

$$
\mathbf{L}_{\mathbf{q}} \equiv \mathbf{q}+i \mathbf{F}_{\mathrm{ext}}, \quad \mathbf{R}_{\mathbf{q}} \equiv \mathbf{q}
$$

A tensorial mobility kernel (19) makes it possible to discuss the motion parallel and perpendicular to the force differently.

\section{B. Irreducible memory function}

A standard step before introducing closure approximations for memory functions is the construction of the so-called irreducible friction kernel [28,29].

We start by writing the adjoint Smoluchowski operator $\Omega^{\dagger}$ as an arbitrary splitting,

$$
\Omega^{\dagger}=\Omega^{\dagger}-\Omega_{0}^{\dagger}+\Omega_{0}^{\dagger},
$$

so that the symmetrized $Q_{\mathrm{s}}$-projected operator takes the form

$$
Q_{\mathrm{s}} \Omega^{\dagger} Q_{\mathrm{s}}=\Omega_{\mathrm{irr}}^{\dagger}+Q_{\mathrm{s}} \Omega_{0}^{\dagger} Q_{\mathrm{s}},
$$

where we have defined

$$
\Omega_{\mathrm{irr}}^{\dagger} \equiv Q_{\mathrm{s}}\left[\Omega^{\dagger}-\Omega_{0}^{\dagger}\right] Q_{\mathrm{s}} .
$$

We follow [28,29] and set

$$
\Omega_{0}^{\dagger}=-\left|\mathbf{H}_{\mathbf{q}}^{\mathrm{s}}\right\rangle \cdot\left\langle\mathbf{H}_{\mathbf{q}}^{\mathrm{s}}\right| .
$$

We then use the operator identity

$$
e^{(A+B) t}=e^{A t}+\int_{0}^{t} d t^{\prime} e^{A\left(t-t^{\prime}\right)} B e^{(A+B) t^{\prime}},
$$

with $A=\Omega_{\text {irr }}^{\dagger}$ and $B=Q_{\mathrm{s}} \Omega_{0}^{\dagger} Q_{\mathrm{s}}$, to write the projected timeevolution operator in the form

$e^{Q_{\mathrm{s}} \Omega^{\dagger} Q_{\mathrm{s}} t}=e^{\Omega_{\mathrm{irr}}^{\dagger} t}-\int_{0}^{t} d t^{\prime} e^{\Omega_{\mathrm{irr}}^{\dagger}\left(t-t^{\prime}\right)}\left|\mathbf{H}_{\mathbf{q}}^{\mathrm{s}}\right\rangle \cdot\left\langle\mathbf{H}_{\mathbf{q}}^{\mathrm{s}}\right| e^{Q_{\mathrm{s}} \Omega^{\dagger} Q_{\mathrm{s}} t^{\prime}}$.

Taking the elements $\left\langle\mathbf{H}_{\mathbf{q}}^{\mathrm{s}}|\cdots| \mathbf{H}_{\mathbf{q}}^{\mathrm{s}}\right\rangle$ of Eq. (26) and using Eq. (19) yields a tensor integral equation

$$
\mathbf{M}_{\mathbf{q}}(t)=\mathbf{m}_{\mathbf{q}}(t)-\int_{0}^{t} \mathbf{m}_{\mathbf{q}}\left(t-t^{\prime}\right) \cdot \mathbf{M}_{\mathbf{q}}\left(t^{\prime}\right) d t^{\prime},
$$

relating the tensor mobility kernel $\mathbf{M}_{\mathbf{q}}(t)$ with the so-called irreducible friction tensor kernel, defined by

$$
\mathbf{m}_{\mathbf{q}}(t)=\left\langle\mathbf{H}_{\mathbf{q}}^{\mathrm{s}} \mid e^{\Omega_{\mathrm{irr}}^{\dagger} t} \mathbf{H}_{\mathbf{q}}^{\mathrm{s}}\right\rangle .
$$

While, in general, the friction kernel for the transient time dependence will depend on the two times $t$ and $t^{\prime}$ in Eq. (27) independently (see, e.g., [7]), in the present case of constant external force, the dependence simplifies and only the time difference enters.

It is well established [6] from experience with quiescent MCT that the irreducible friction kernel is better suited to mode-coupling type of approximation than the mobility kernel. We then close the set of equations for the probe correlator $\Phi_{\mathbf{q}}^{\mathrm{s}}(t)$ via the friction kernel $\mathbf{m}_{\mathbf{q}}(t)$ by writing it in terms of $\Phi_{\mathbf{q}}^{\mathrm{s}}(t)$ in functional form

$$
\mathbf{m}_{\mathbf{q}}(t)=\mathscr{C}_{\mathbf{q}}\left[\boldsymbol{\Phi}^{\mathrm{s}}(t) ; \boldsymbol{\Phi}(t)\right]
$$

The relation above formally couples $\mathbf{m}_{\mathbf{q}}(t)$ to the dynamics of the host system, described by $\Phi_{q}(t)$.

Previous MCT approaches to microrheology [17,18,20] were based on a single relaxation channel, described by a scalar irreducible kernel. It was derived from the scalar mobility kernel $M_{\mathbf{q}}(t)$ in its original microscopic form (14), i.e., as a dynamical correlation of the object $A_{\mathbf{q}}^{\mathrm{s}}$. The latter may be interpreted as the force on the probe, which includes a fast fluctuating part due to the host particles and also a deterministic component, due to the external force. The anisotropic character of the problem entered the description solely via the dependency of correlators and memory functions on the direction of the wave vector $\mathbf{q}$ (and not only on its modulus).

In the present treatment the deterministic force on the probe has been separated out [Eq. (17)] from the stochastic components. The irreducible friction kernel is generated from the tensor mobility kernel $\mathbf{M}_{\mathbf{q}}(t)$, both being dynamical correlation functions of the strictly fluctuating force $\mathbf{H}_{\mathbf{q}}^{\mathrm{s}}$ on the probe (with the respective time-evolution operator). Anisotropy arising from the external driving manifests itself in the tensor character of the microscopic correlations underlying $\mathbf{M}_{\mathbf{q}}(t)$ and $\mathbf{m}_{\mathbf{q}}(t)$. This approach gives rise to multiple decay channels [24,25], corresponding to different matrix elements of $\mathbf{m}_{\mathbf{q}}(t)$. 
For the anisotropic microrheology problem, memory functions arising from tensor correlations of $\mathbf{H}_{\mathbf{q}}^{\mathrm{s}}$ are more amenable to mode-coupling closure approximations than those representing scalar dynamic correlations of the variable $A_{\mathbf{q}}^{\mathrm{s}}$. We do not try to a priori substantiate this conclusion. It was reached a posteriori, based on the stability of the numerical solutions of the equations of motion for $\Phi_{\mathbf{q}}^{\mathrm{s}}(t)$ that resulted from each approach (single and multiple relaxation channels).

\section{Effective friction kernel}

Calculation of the probe correlator $\Phi_{\mathbf{q}}^{\mathrm{s}}(t)$ formally requires the solution of the set of coupled equations (12) and (27) supplemented by the closure relation (29). In this section we recast this set of equations into the simpler form (4). This leads to the definition of an effective scalar memory kernel [30].

With the help of the Laplace transform, defined for a timedependent quantity $A(t)$ by

$$
\tilde{A}(s)=\int_{0}^{\infty} d t e^{-s t} A(t),
$$

Eqs. (12) and (27) may be combined into the form

$$
\tilde{\Phi}_{\mathbf{q}}^{\mathrm{s}}(s)=\frac{1}{s+\mathbf{L}_{\mathbf{q}}^{*} \cdot\left[\mathbf{1}+\tilde{\mathbf{m}}_{\mathbf{q}}\right]^{-1} \cdot \mathbf{R}_{\mathbf{q}}},
$$

where $\tilde{\Phi}_{\mathbf{q}}^{\mathrm{s}}(s)$ and $\tilde{\mathbf{m}}_{\mathbf{q}}(s)$ are the Laplace transforms of $\Phi_{\mathbf{q}}^{\mathrm{s}}(t)$ and $\mathbf{m}_{\mathbf{q}}(t)$, respectively.

We define the effective friction kernel $\tilde{m}_{\mathbf{q}}(s)$ through

$$
\mathbf{L}_{\mathbf{q}}^{*} \cdot\left[\mathbf{1}+\tilde{\mathbf{m}}_{\mathbf{q}}\right]^{-1} \cdot \mathbf{R}_{\mathbf{q}} \equiv \frac{\Gamma_{\mathbf{q}}}{1+\tilde{m}_{\mathbf{q}}},
$$

so that Eq. (31) may be recast into the simple form

$$
\tilde{\Phi}_{\mathbf{q}}^{\mathrm{s}}(s)=\frac{1}{s+\frac{\Gamma_{\mathbf{q}}}{1+\tilde{m}_{q}}} .
$$

Equation (33) is the Laplace counterpart of Eq. (4), where $\tilde{m}_{\mathbf{q}}(s)$ is the Laplace transform of the effective friction kernel $m_{\mathbf{q}}(t)$.

In Appendix A we show that the probe correlator exhibits cylindrical symmetry, viz. $\tilde{\Phi}_{\mathbf{q}}^{\mathrm{s}}(s)=\tilde{\Phi}_{Q \mathbf{q}}^{\mathrm{s}}(s)$ holds for any rotation matrix $Q$ with $Q \hat{\mathbf{z}}=\hat{\mathbf{z}}$. With the help of Eq. (31) one can deduce that cylindrically symmetry of $\tilde{\Phi}^{\mathrm{s}}$ holds if and only if the tensor friction kernel obeys

$$
\tilde{\mathbf{m}}_{\mathbf{q}}=Q^{T} \tilde{\mathbf{m}}_{Q \mathbf{q}} Q .
$$

Hence, we can express the tensor for any direction $\mathbf{q}$ in terms of the tensor for a direction $\overline{\mathbf{q}}$ in a plane, which is spanned by one vector parallel to the force and one vector perpendicular to the force by simply rotating it into this plane via $Q$. We choose the $x z$ plane, i.e., $\overline{\mathbf{q}}=\left(q_{x}, 0, q_{z}\right)$.

For this particular choice, the elements $\tilde{m}_{\overline{\mathbf{q}}}^{x y}$ and $\tilde{m}_{\overline{\mathbf{q}}}^{y z}$ reduce to zero. This can be seen by looking at the behavior under the transformation $y_{1} \mapsto-y_{1}$ of the integrand in the inner product in Eq. (28), where $y_{1}$ is the $y$ coordinate of the probe particle. The tensor friction kernel $\tilde{\mathbf{m}}_{\overline{\mathbf{q}}}$ then has a simple matrix representation [31],

$$
\tilde{\mathbf{m}}_{\overline{\mathbf{q}}}=\left(\begin{array}{ccc}
\tilde{m}_{\overline{\mathbf{q}}}^{x x} & 0 & \tilde{m}_{\overline{\mathbf{q}}}^{x z} \\
0 & \tilde{m}_{\overline{\mathbf{q}}}^{y y} & 0 \\
\tilde{m}_{\overline{\mathbf{q}}}^{x z} & 0 & \tilde{m}_{\overline{\mathbf{q}}}^{z z}
\end{array}\right) .
$$

Thus, the object $\left[\mathbf{1}+\tilde{\mathbf{m}}_{\mathbf{q}}\right]^{-1}$ in Eq. (32) has a simple closed form. Making use of the cylindrical symmetry by calculating the correlation function only for $\overline{\mathbf{q}}$, the rotation matrix $Q$ in Eq. (34) reduces to the identity and thus the left-hand side of (32) may be readily evaluated to provide an exact expression for $\tilde{m}_{\mathbf{q}}(s)$ in terms of three elements of $\tilde{\mathbf{m}}_{\mathbf{q}}$,

$$
\frac{\Gamma_{\mathbf{q}}}{1+\tilde{m}_{\mathbf{q}}}=\frac{\Gamma_{\mathbf{q}}^{x}\left(1+\tilde{m}_{\mathbf{q}}^{z z}\right)-\Gamma_{\mathbf{q}}^{x z} \tilde{m}_{\mathbf{q}}^{x z}+\Gamma_{\mathbf{q}}^{z}\left(1+\tilde{m}_{\mathbf{q}}^{x x}\right)}{\left(1+\tilde{m}_{\mathbf{q}}^{x x}\right)\left(1+\tilde{m}_{\mathbf{q}}^{z z}\right)-\tilde{m}_{\mathbf{q}}^{x z} \tilde{m}_{\mathbf{q}}^{x z}},
$$

where

$$
\Gamma_{\mathbf{q}}=\mathbf{L}_{\mathbf{q}}^{*} \cdot \mathbf{R}_{\mathbf{q}}=q_{x}^{2}+q_{z}^{2}-i q_{z} F
$$

and

$$
\begin{aligned}
\Gamma_{\mathbf{q}}^{x} & =q_{x}^{2}, \\
\Gamma_{\mathbf{q}}^{z} & =q_{z}^{2}-i q_{z} F, \\
\Gamma_{\mathbf{q}}^{x z} & =2 q_{x} q_{z}-i q_{x} F .
\end{aligned}
$$

In the time domain, Eq. (36) yields an integral equation for the effective kernel $m_{\mathbf{q}}(t)$,

$$
\begin{aligned}
m_{\mathbf{q}}(t) & +\int_{0}^{t} m_{\mathbf{q}}\left(t-t^{\prime}\right) \alpha_{\mathbf{q}}\left(t^{\prime}\right) d t^{\prime} \\
= & \beta_{\mathbf{q}}(t)+\int_{0}^{t} m_{\mathbf{q}}^{x x}\left(t-t^{\prime}\right) m_{\mathbf{q}}^{z z}\left(t^{\prime}\right) d t^{\prime} \\
& -\int_{0}^{t} m_{\mathbf{q}}^{x z}\left(t-t^{\prime}\right) m_{\mathbf{q}}^{x z}\left(t^{\prime}\right) d t^{\prime},
\end{aligned}
$$

with

$$
\alpha_{\mathbf{q}}(t) \equiv \frac{\Gamma_{\mathbf{q}}^{z}}{\Gamma_{\mathbf{q}}} m_{\mathbf{q}}^{x x}(t)+\frac{\Gamma_{\mathbf{q}}^{x}}{\Gamma_{\mathbf{q}}} m_{\mathbf{q}}^{z z}(t)-\frac{\Gamma_{\mathbf{q}}^{x z}}{\Gamma_{\mathbf{q}}} m_{\mathbf{q}}^{x z}(t)
$$

and

$$
\beta_{\mathbf{q}}(t) \equiv \frac{\Gamma_{\mathbf{q}}^{x}}{\Gamma_{\mathbf{q}}} m_{\mathbf{q}}^{x x}(t)+\frac{\Gamma_{\mathbf{q}}^{z}}{\Gamma_{\mathbf{q}}} m_{\mathbf{q}}^{z z}(t)+\frac{\Gamma_{\mathbf{q}}^{x z}}{\Gamma_{\mathbf{q}}} m_{\mathbf{q}}^{x z}(t) .
$$

So far we have performed only formal manipulations under the sole assumption of axial symmetry. It remains to elaborate a mode-coupling approximation for the functional (29).

\section{Mode-coupling approximation}

The program to construct a mode-coupling approximation to the functional (29) starts by writing

$$
\mathbf{m}_{\mathbf{q}}(t) \approx\left\langle\mathbf{H}_{\mathbf{q}}^{\mathrm{s}} \mid P_{2} e^{\Omega_{\mathrm{irr}}^{\dagger} t} P_{2} \mathbf{H}_{\mathbf{q}}^{\mathrm{s}}\right\rangle,
$$

where the irreducible dynamics has been projected by the operator

$$
P_{2}=\sum_{\mathbf{k}, \mathbf{p}}\left|\rho_{\mathbf{k}}^{\mathrm{s}} \rho_{\mathbf{p}}\right\rangle \frac{1}{N S_{p}}\left\langle\rho_{\mathbf{k}}^{\mathrm{s}} \rho_{\mathbf{p}}\right|
$$

into the space spanned by the product $\rho_{\mathbf{k}}^{\mathrm{s}} \rho_{\mathbf{p}}$ of host and probeparticle density fluctuations. Here $\rho_{\mathbf{q}}=\sum_{j=2}^{N} \exp \left[i \mathbf{q} \cdot \mathbf{r}_{j}\right]$ is the Fourier transform of the host density $\rho(\mathbf{r})=\sum_{j=2}^{N} \delta\left(\mathbf{r}-\mathbf{r}_{j}\right)$, and

$$
S_{q}=\frac{1}{N}\left\langle\rho_{\mathbf{q}} \mid \rho_{\mathbf{q}}\right\rangle
$$


is the equilibrium static structure factor of the $N$-particle host system. Note that $P_{2}$ given by Eq. (45) is the lowest-order projector using the densities $\rho_{\mathbf{p}}$ and $\rho_{\mathbf{k}}^{\mathbf{s}}$ that gives a finite overlap.

The projection in Eq. (44) generates a four-point correlation function. The next approximation step is to factorize the latter into a product of two-point correlation functions and at the same time replace the irreducible time evolution operator $e^{\Omega_{\mathrm{irr}}^{\dagger} t}$ by the original one $e^{\Omega^{\dagger} t}$, viz.

$$
\begin{aligned}
\left\langle\rho_{\mathbf{k}}^{\mathrm{s}} \rho_{\mathbf{p}} \mid e^{\Omega_{\mathrm{rrr}}^{\dagger} t} \rho_{\mathbf{k}^{\prime}}^{\mathrm{s}} \rho_{\mathbf{p}^{\prime}}\right\rangle & \approx\left\langle\rho_{\mathbf{k}}^{\mathrm{s}} \mid e^{\Omega^{\dagger} t} \rho_{\mathbf{k}^{\prime}}^{\mathrm{s}}\right\rangle\left\langle\rho_{\mathbf{p}} \mid e^{\Omega^{\dagger} t} \rho_{\mathbf{p}^{\prime}}\right\rangle \\
& =\delta_{\mathbf{k}, \mathbf{k}^{\prime}} \delta_{\mathbf{p}, \mathbf{p}^{\prime}} \Phi_{\mathbf{k}}^{\mathrm{s}}(t) N S_{p} \Phi_{\mathbf{p}}(t),
\end{aligned}
$$

where

$$
\Phi_{\mathbf{q}}(t)=\frac{1}{N S_{q}}\left\langle\rho_{\mathbf{q}} \mid e^{\Omega^{\dagger} t} \rho_{\mathbf{q}}\right\rangle
$$

is the $(N$-particle) intermediate scattering function of the host system or simply the host correlator. We note that the normalization of the projection operator $P_{2}$ in (45) consistently incorporates the factorization (47) taken at $t=0$.

Substitution of (47) into (44) yields

$$
\mathbf{m}_{\mathbf{q}}(t) \approx \sum_{\mathbf{k}, \mathbf{p}} \frac{\Phi_{\mathbf{k}}^{\mathrm{s}}(t) \Phi_{\mathbf{p}}(t)}{N S_{p}}\left\langle\mathbf{H}_{\mathbf{q}}^{\mathrm{s}} \mid \rho_{\mathbf{k}}^{\mathrm{s}} \rho_{\mathbf{p}}\right\rangle\left\langle\rho_{\mathbf{k}}^{\mathrm{s}} \rho_{\mathbf{p}} \mid \mathbf{H}_{\mathbf{q}}^{\mathrm{s}}\right\rangle .
$$

To obtain explicit expressions, we evaluate (details in Refs. [18,31])

$$
\left\langle\mathbf{H}_{\mathbf{q}}^{\mathrm{s}} \mid \rho_{\mathbf{k}}^{\mathrm{s}} \rho_{\mathbf{p}}\right\rangle=\left\langle\rho_{\mathbf{k}}^{\mathrm{s}} \rho_{\mathbf{p}} \mid \mathbf{H}_{\mathbf{q}}^{\mathrm{s}}\right\rangle^{*}=i(\mathbf{q}-\mathbf{k}) S_{p}^{\mathrm{s}} \delta_{\mathbf{q}-\mathbf{k}, \mathbf{p}},
$$

where

$$
S_{q}^{\mathrm{s}}=\left\langle\rho_{\mathbf{q}}^{\mathrm{s}} \mid \rho_{\mathbf{q}}\right\rangle
$$

is the probe-host static structure factor. For the present case, where the probe is identical to the host particles, $S_{q}^{\mathrm{s}}$ is connected to the static structure factor by $S_{q}^{\mathrm{s}}=S_{q}-1$.

Substituting Eq. (50) into Eq. (49) and taking the thermodynamic limit for large volume $V$ with

$$
\lim _{V \rightarrow \infty} \frac{1}{V} \sum_{\mathbf{k}}=\frac{1}{(2 \pi)^{3}} \int d \mathbf{k}
$$

the kernel (49) becomes

$$
\mathbf{m}_{\mathbf{q}}(t) \approx \frac{1}{(2 \pi)^{3}} \int d \mathbf{k p p} \frac{\left(S_{p}^{\mathrm{s}}\right)^{2}}{n S_{p}} \Phi_{\mathbf{k}}^{\mathrm{s}}(t) \Phi_{p}(t),
$$

where $\mathbf{p}=\mathbf{q}-\mathbf{k}$ and $\mathbf{p p}$ denotes a dyadic product. Here $S_{q}, \Phi_{q}(t)$, and $S_{q}^{\mathrm{s}}$ are the infinite-system counterparts of the $N$-particle quantities defined in Eqs. (46), (48), and (51), respectively. Appendix B contains the axisymmetric form of the MCT friction kernel (52), which is suitable for numerical calculations.

The equilibrium static structure factor $S_{q}$ can be obtained from liquid state theory [32]. In this work it is calculated for a fluid of hard spheres via the Percus-Yevick expression for a given volume fraction $\phi$ of the host system, where $\phi=\frac{4 \pi}{3}\left(\frac{d}{2}\right)^{3} \frac{N}{V}$. The equilibrium $S_{q}$ also specifies the host correlator $\Phi_{q}(t)$, which is precalculated according to the algorithm presented in [33] for quiescent MCT.
In the absence of an external force it can be shown [31] that (52) reduces to the isotropic MCT friction kernel for quiescent tagged-particle motion presented in Ref. [12]. For finite external forces, the relations (34) (cumbersome [31]) and (35) [easy; see Eq. (52)] can be verified, which shows that MCT is consistent with cylindrical symmetry.

Summarizing, we have arrived at a closed set of equations for explicit calculations of the probe correlator $\Phi_{\mathbf{q}}^{\mathrm{s}}(t)$. This is a fully microscopic MCT model for colloidal hard spheres with a predictive character and two control parameters, $\phi$ and $F$.

Note that the MCT functional (52) for the tensor friction kernel is local in time; that is, $\mathbf{m}_{\mathbf{q}}(t)$ is determined by the correlators $\Phi_{\mathbf{q}}^{\mathrm{s}}(t)$ and $\Phi_{q}(t)$ at the same time argument $t$. This is not the case of the effective friction kernel $m_{\mathbf{q}}(t)$, which implicitly depends on correlators at times $t^{\prime}<t$ through the integral equation (41). Its general properties were discussed for MCT equations containing only real-valued coefficients [25].

The numerical scheme to solve (4) for $\Phi_{\mathbf{q}}^{\mathrm{s}}(t)$ with a nonlocal (in time) memory kernel is outlined in the Appendixes $\mathrm{C}$ and D. Accuracy tests of the numerical solutions are presented in Appendix E.

\section{E. Delocalization transition of the probe}

Ideal localized states, where the probe particle is trapped by its surrounding host particles, are characterized by nonvanishing probe nonergodicity parameters,

$$
f_{\mathbf{q}}^{\mathrm{s}}=\lim _{t \rightarrow \infty} \Phi_{\mathbf{q}}^{\mathrm{s}}(t)=\lim _{s \rightarrow 0} s \tilde{\Phi}_{\mathbf{q}}^{\mathrm{s}}(s) .
$$

Equation (33) yields

$$
f_{\mathbf{q}}^{\mathrm{s}}=\frac{1}{1+\frac{\Gamma_{\mathbf{q}}}{m_{\mathbf{q}}(\infty)}}
$$

where $m_{\mathbf{q}}(\infty)$ is the long-time limit of the effective friction kernel,

$$
m_{\mathbf{q}}(\infty)=\lim _{t \rightarrow \infty} m_{\mathbf{q}}(t)=\lim _{s \rightarrow 0} s \tilde{m}_{\mathbf{q}}(s)
$$

It is related to the long-time limit $\boldsymbol{\mu}_{\mathbf{q}}$ of $\mathbf{m}_{\mathbf{q}}(t)$, viz.

$$
\boldsymbol{\mu}_{\mathbf{q}}=\lim _{t \rightarrow \infty} \mathbf{m}_{\mathbf{q}}(t)=\lim _{s \rightarrow 0} s \tilde{\mathbf{m}}_{\mathbf{q}}(s),
$$

through Eq. (32),

$\frac{\Gamma_{\mathbf{q}}}{m_{\mathbf{q}}(\infty)}=\mathbf{L}_{\mathbf{q}}^{*} \cdot \boldsymbol{\mu}_{\mathbf{q}}^{-1} \cdot \mathbf{R}_{\mathbf{q}}=\frac{\Gamma_{\mathbf{q}}^{x} \mu_{\mathbf{q}}^{z z}+\Gamma_{\mathbf{q}}^{z} \mu_{\mathbf{q}}^{x x}-\Gamma_{\mathbf{q}}^{x z} \mu_{\mathbf{q}}^{x z}}{\mu_{\mathbf{q}}^{x x} \mu_{\mathbf{q}}^{z z}-\left(\mu_{\mathbf{q}}^{x z}\right)^{2}}$.

Equations (54) and (55) imply that localized states of the probe entail the arrest of the effective friction kernel. This is independent of the mode-coupling approximation.

The approximation (52) leads to

$$
\boldsymbol{\mu}_{\mathbf{q}} \approx \frac{1}{(2 \pi)^{3}} \int d \mathbf{k p p} \frac{\left(S_{p}^{\mathrm{s}}\right)^{2}}{n S_{p}} f_{p} f_{\mathbf{k}}^{\mathrm{s}}
$$

so that localized states of the probe require the host system to be in the ideal glass state with arrested host correlator $f_{q}=\lim _{t \rightarrow \infty} \Phi_{q}(t)$. Within the assumed quiescent MCT dynamics for the host [33], ideal glass states occur for $\phi>\phi_{g}^{\mathrm{MCT}} \approx 0.5159$. 


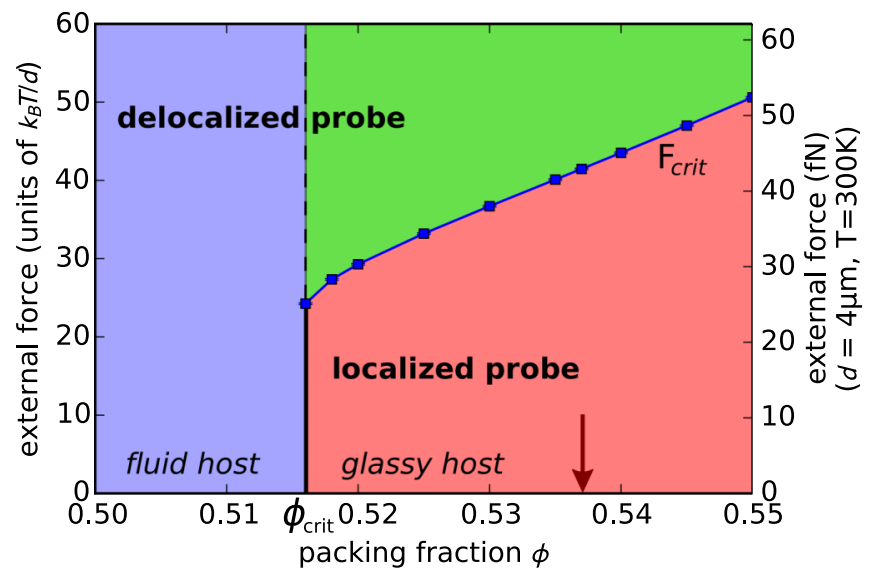

FIG. 1. MCT phase diagram separating delocalized and ideal localized regimes. The vertical arrow marks the density chosen in the MCT calculations for the comparison with simulations. The right vertical axis gives forces in real units converted assuming colloids of size $d=4 \mu \mathrm{m}$ close to room temperature.

For given values of the control parameters $\left(F, \phi>\phi_{g}^{\mathrm{MCT}}\right)$, the host nonergodicity parameter $f_{q}$ is calculated independently [33] and Eq. (54) [supplemented by (57) and (58)] may be solved by iteration using $f_{\mathbf{q}}^{\text {s }}=1$ as starting values. For forces $F$ on the probe smaller than a threshold $F_{c}$, the probe particle remains localized and the iterative solution of (54) provides nonvanishing values for $f_{\mathbf{q}}^{\mathrm{s}}$. Forces $F>F_{c}$ delocalize the probe and drag it through the arrested host. This delocalization transition is signaled by the vanishing of $f_{\mathbf{q}}^{\mathrm{s}}$.

We are then able to calculate the critical force $F_{c}$ as a function of the volume fraction $\phi$ of the host, depicted in Fig. 1. This is a MCT phase diagram separating mobile (or delocalized) from ideal localized states of the probe. For $\phi<\phi_{g}^{\mathrm{MCT}}, F_{c}=0$, as the probe cannot be trapped if the host particles are in the fluid state. While comparison with existing experimental data on glassy colloids is difficult because a probe twice larger than the bath particles was used [34], the order of magnitude of the critical force appears accessible to experiments on colloidal dispersions [35] or microemulsions [36]; see the right force axis in Fig. 1, where actual force values are given for particles of size $d=4 \mu \mathrm{m}$.

The critical force as a function of $\phi$ presented in Fig. 1 can be directly compared with the rescaled value $\phi^{-2 / 3} \tau_{\mathrm{abs}}$ of the dimensionless absolute yield stress $\tau_{\text {abs }}$ predicted by the isotropic nonlinear Langevin equation (NLE) approach [23]. The rescaling comes from the assumption [23] that an applied macroscopic stress is transmitted to the particle level via a microscopic force acting on the tagged particle. The NLE data for $\tau_{\text {abs }}$ can be fitted [23] by a high-power-law $\phi$ dependence, $\tau_{\text {abs }} \sim 17200 \phi^{11}$. At a volume fraction $\phi=0.516 \approx \phi_{g}^{\mathrm{MCT}}$, NLE predicts a critical force $\sim 19 k_{\mathrm{B}} T / d$, lower than our result of $\sim 24 k_{\mathrm{B}} T$. At the volume fraction $\phi=0.537$ chosen for the present MCT calculations, NLE yields $F_{c} \sim 28 k_{\mathrm{B}} T / d$, smaller than the result $F_{c} \sim 42 k_{\mathrm{B}} T / d$ predicted by our theory.

\section{SIMULATIONS}

In the simulations, a polydisperse system of quasihard spherical particles is considered. All particles follow Langevin dynamics, which for particle $j$ is given by the Langevin equation of motion [27],

$$
m \ddot{\mathbf{r}}_{j}=\sum_{i} \mathbf{F}_{i j}-\gamma_{0} \dot{\mathbf{r}}_{j}+\eta_{j}(t)+\mathbf{F}_{\mathrm{ext}} \delta_{j 1},
$$

where the direct interactions, $\mathbf{F}_{i j}$, derive from an inversepower potential: $U_{i j}(r)=k_{\mathrm{B}} T\left(r / d_{i j}\right)^{-36}$, with $d_{i j}$ the centerto-center distance between particles, $\gamma_{0} \dot{\mathbf{r}}_{j}$ the friction with the solvent, and $\boldsymbol{\eta}_{j}$ the random force, and the external force $\mathbf{F}_{\text {ext }}=F \hat{\mathbf{z}}$ is applied only to the probe (labeled 1). The friction and random forces are linked by the fluctuation-dissipation theorem.

The simulations are run in a cubic box, with $N=1000$ particles and periodic boundary conditions. All particles have the same mass, $m=1$; diameters, $d_{i}$, are distributed according to a flat distribution centred around $d=1$ of width $\delta=0.1 d$; and the thermal energy is $k_{\mathrm{B}} T=1$. The solvent friction coefficient is set $\gamma_{0}=10 \sqrt{m k_{\mathrm{B}} T} / d$, giving a diffusion coefficient of the free particle $D_{0}=k_{\mathrm{B}} T / \gamma_{0}=0.1 d \sqrt{k_{\mathrm{B}} T / m}$. Time is measured in units of the Newtonian microscopic time $d \sqrt{m / k_{\mathrm{B}} T}$, but converted to Brownian units using the Brownian time scale, $\tau_{\mathrm{B}}=d^{2} / D_{0}$. The equations of motion are integrated using a Heun algorithm with a time step of $0.00025 d \sqrt{m / k_{\mathrm{B}} T}=2.5 \times 10^{-5} \tau_{\mathrm{B}}$ [37].

For microrheology, a particle is randomly selected as probe. At $t=0$, it is pulled with a constant force through the system, and its trajectory is monitored as a function of time; the results, therefore, comprise the transient regime. The probe is allowed to travel through the simulation box more than once, as we could not identify any different behavior between the first and consecutive passages. The results presented below are the average over ca. 2500 independent trajectories (probes) for every force. A snapshot of the system is presented in Fig. 2 with the probe marked in red and the particles in front of it removed to allow seeing it.

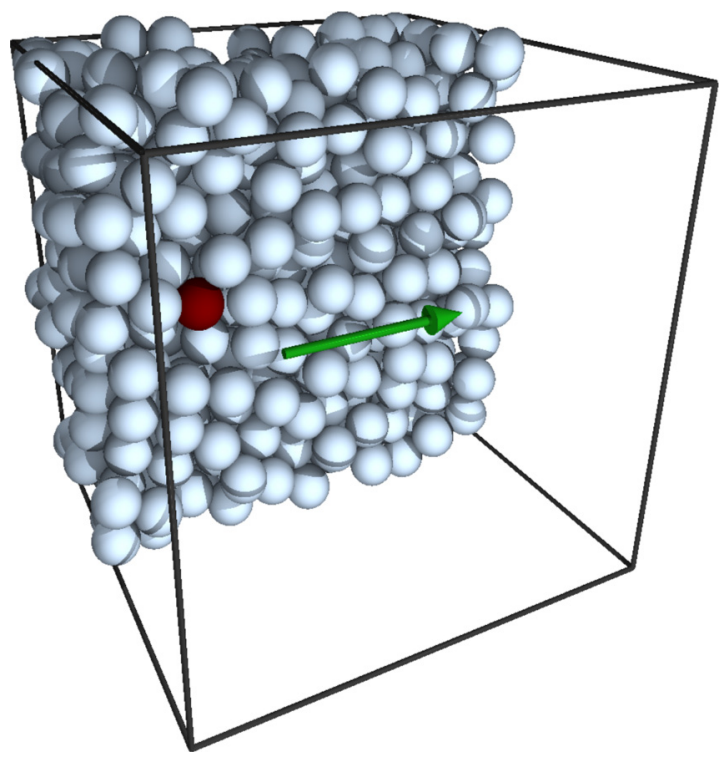

FIG. 2. Snapshot of the system with the probe marked in red (dark gray) and all particles in front of it removed to allow observing the probe. The arrow indicates the external force applied only to the probe. 


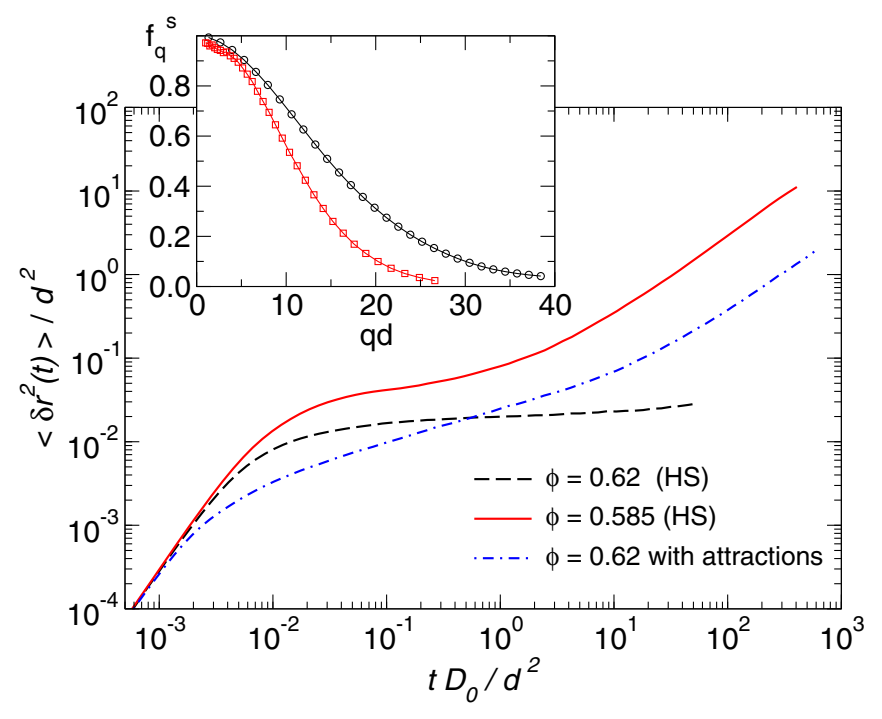

FIG. 3. Mean-squared displacement of the bulk system with $\phi=0.62$ (black dashed line), compared with the system with attractions and the same density, in the fluid region (blue dash-dotted line), and with the MSD of quasihard spheres at a lower density, $\phi=0.585$, again in the fluid (red solid line). (Inset) Nonergodicity parameters of the system with $\phi=0.62$ (circles) and the critical packing fraction for the glass transition (squares).

The volume fraction occupied by the particles is constant, $\phi=0.62$, calculated considering polydisperse hard spheres of diameter $d_{i}$. This volume fraction corresponds to a state in the glassy region, as estimated by an MCT analysis for this system, that yielded the glass transition at $\phi_{g}=0.596[38,39]$. In order to prepare the system at this volume fraction, without introducing large local stresses, we add a short-range attraction to the interaction potential [13]. It is well known that the addition of short-range attractions to purely repulsive interactions increases the glass transition density for moderate attraction strengths, whereas intense attractions induce an attraction driven glass transition at all densities [40].

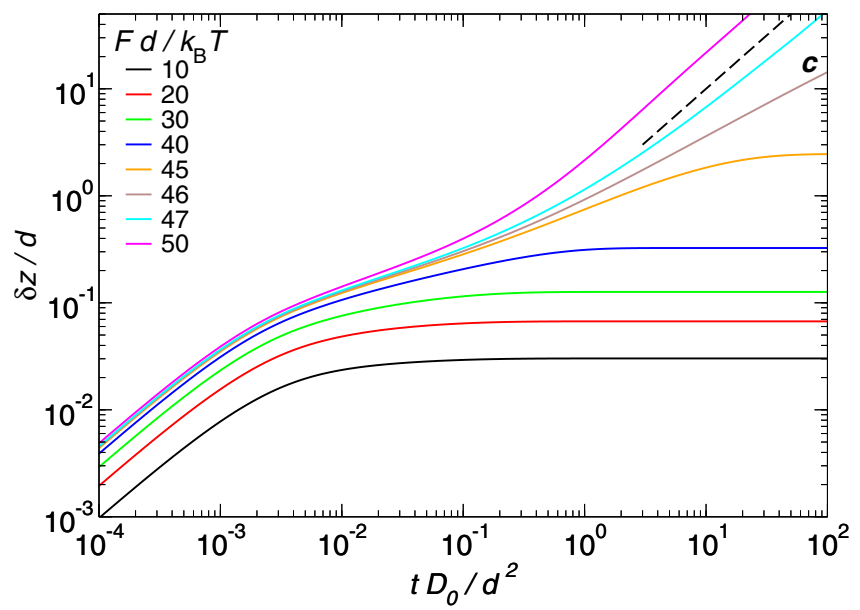

We use as attractive contribution the Asakura-Oosawa interaction with a minimum of $4 k_{\mathrm{B}} T$ and a range $\xi=0.10 d$, added to the quasi-hard-sphere interaction potential [13]. The system with the attractive interaction and a density $\phi=0.62$ is in a fluid state and can be equilibrated, and the final system of quasihard spheres is finally prepared removing the interactions instantly and aging the system. The aging runs for $t_{w}=5000 d \sqrt{m / k_{\mathrm{B}} T}$ with Newtonian dynamics, and further $t_{w}=10000 d \sqrt{m / k_{\mathrm{B}} T}$ with Langevin dynamics.

The mean-squared displacement (MSD) of the equilibrated attractive system and the final glass (after aging) are compared in Fig. 3; a supercooled fluid close to the glass transition, with $\phi=0.585$ (in equilibrium), is also included for comparison. Note that the system with attractions shows shorter localization length than the supercooled HS fluid, due to the short-range attractions, but particles can diffuse over long distances. The system with $\phi=0.62$ without attractions shows no reminiscence of this short localization length, but the cage size is given by the core hindrance. This is further tested by the incoherent nonergodicity parameters, $f_{q}^{s}$, calculated as the height of the self part of the density correlation function. This is shown in the inset, comparing the results for $\phi=0.62$ and at the transition. As expected, in the denser system the particles are more tightly caged, resulting in a shorter localization length and thus higher $f_{q}^{s}$.

\section{RESULTS AND DISCUSSIONS}

Having described in detail our theoretical and simulation schemes to the study of active microrheology, we now present a comparison of the results obtained by both approaches with the aim of assessing the quality of the MCT description.

In colloidal glasses, the microscopic dynamics of single particles is arrested due to the steric hindrance that confines particles in cages formed by their own neighbors. As a result, colloidal glasses exhibit in bulk rheology a yield stress; namely, a finite stress is needed to make them flow. The analog in microrheology is the existence of a critical force, $F_{c}$, [17]; small forces cannot free the probe from its cage of

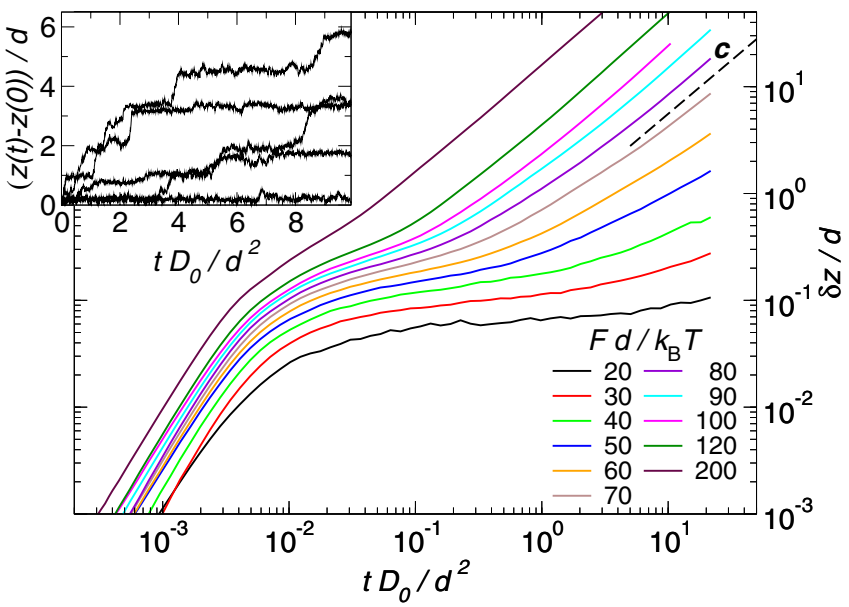

FIG. 4. Probe-particle displacement in the direction of the applied force for different external forces, as labeled. (Left) Theoretical results for a glassy host at volume fraction $\phi=0.537$. (Right) Simulation results for $\phi=0.62$. The curves with the label $c$ correspond to the critical force in each case. The dashed line shows the $\delta z \propto t$ behavior. In both graphs the force increases from bottom to top. The inset shows typical individual probe trajectories for $F=50 k_{\mathrm{B}} T / d$. 
neighbors, whereas stronger ones can make it attain a steady velocity. This is tested in Fig. 4, where the probe displacement in the force direction for increasing external forces is presented.

The theoretical results indeed show a transition from a low-force regime, where the probe is localized (or caged), to a high-force region, where a steady velocity is reached, that grows with the external force. In the localized regime, a sublinear evolution from a shoulder reminiscent of the localization length in the glass to a long-time plateau is observed. This long-time plateau is higher with increasing forces and is reached at longer times, as the transition is reached. The data corresponding to the critical force, labeled with a $c$ in the figure, show a steady sublinear increase. In the simulations, these predictions are partially confirmed; the probe is localized only for small forces, and sublinear behavior with time following the short-time localization is observed at moderate forces, but the long-time plateau is not reached, while a steady velocity is observed at large forces.
Longer simulations, run at particular forces, did not show any indication of a saturation in $\delta z$.

It must be noted that the simulation results are the average over ca. 2500 trajectories. The inset to the figure shows several individual trajectories for $F=50 k_{\mathrm{B}} T / d$, which result in the sublinear increase of $\delta z$ with time. The whole set comprises fully arrested trajectories, and stairslike evolutions; the steps have an amplitude of one diameter approximately, indicating that the probe overtakes the particle in front, switching positions. The sublinear growth of $\delta z$ implies that these steps are less frequent for longer times and are therefore characteristic of the transient regime. Similar phenomenology has been observed previously in simulations of microrheology of a Yukawa mixture [41] and hard-disk glasses [20], with probe trajectories exhibiting intermittent dynamics. This heterogeneity of the trajectories is captured in extremely broad displacement probability distributions predicted by the theory. Yet, it may underestimate the probability of repeated hops of the probe, which can be responsible for some
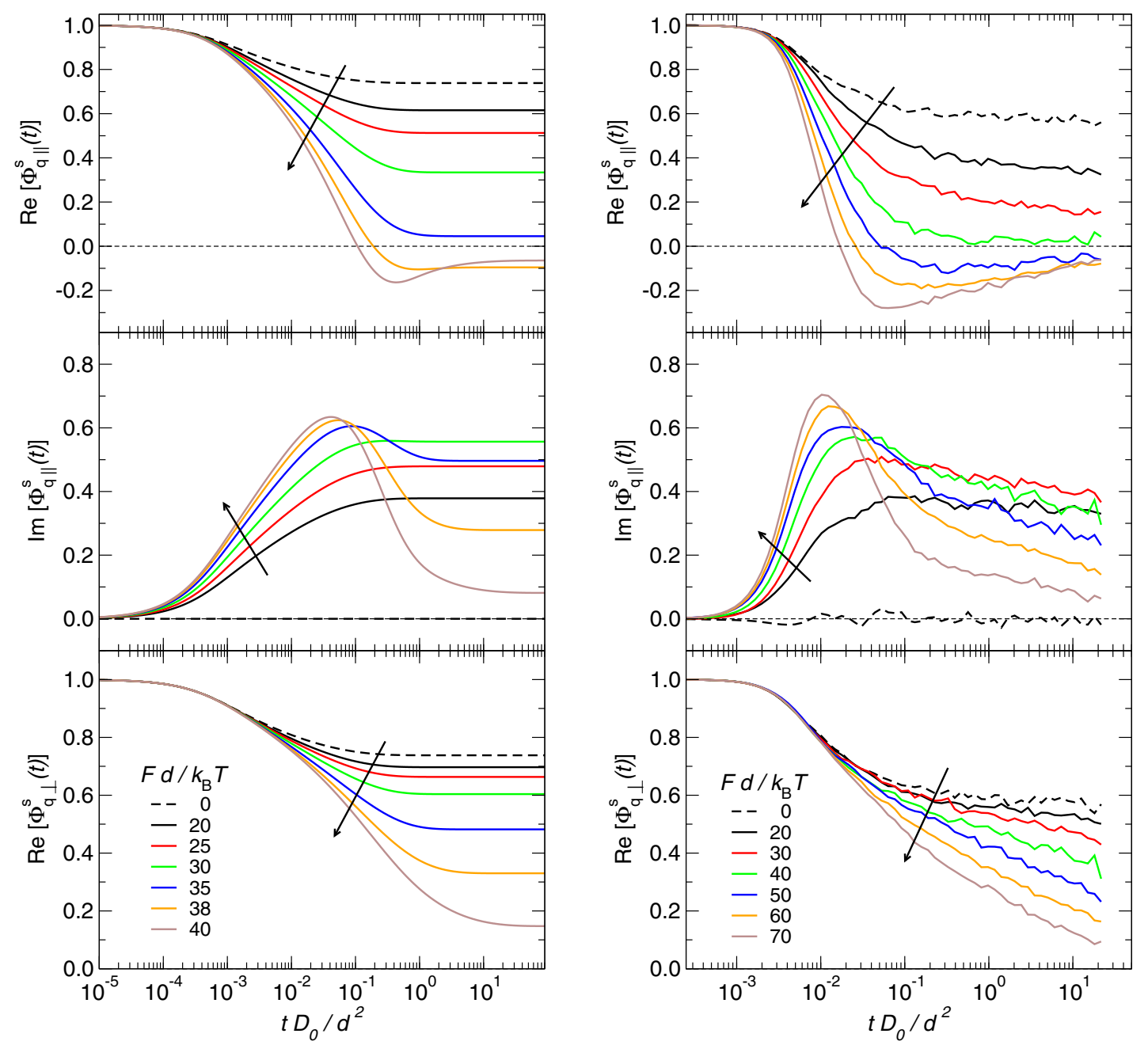

FIG. 5. Probe position correlation functions for different forces in the localized regime, for a wave vector modulus close to the location $q_{2}$, the position of the second peak of the structure factor, and different directions: in the direction of the external force (real and imaginary parts of the correlation function in the top and middle panels, respectively) and perpendicular to the external force (bottom panels). In the theory (left panels), $\phi=0.537$ and the wave vector is $q d=12.75$. In the simulations (right panels), $\phi=0.62$ and the wave vector is $q d=13.35$. The dashed lines in both panels represent the results for the unforced probe, i.e., $F=0 k_{\mathrm{B}} T / d$. The arrows indicate the direction of increasing force. 
differences between the theory and simulations, discussed below, particularly, the absence of a well-defined threshold force in the simulated system.

In the next sections, we will perform a detailed comparison between theory and simulations, focusing on the localized regime. We consider a host volume fraction $\phi=0.537$ in the MCT calculations, to keep approximately the same relative distance $\left(\phi-\phi_{g}\right) / \phi_{g}$ to the glass transition in the host system as in the simulations. (This particular volume fraction was used in the comparison shown in Fig. 4.) We study the dynamics of the probe, shape of the cage, and the properties of the critical force, using the probe position correlation function $\Phi_{\mathbf{q}}^{\mathrm{s}}(t)$ for different wave vectors. Two different numerical discretizations will be employed. A fine one for the evaluation of the static, nonergodic quantities obtained in the long-time limit of Sec. II E, and a coarser one to find the time-dependent correlation functions from Eqs. (12), (27), and (52); see Appendix B for more details.

\section{A. Probe position correlation functions in the localized regime}

Since in the localized regime the probe moves only a fraction of its diameter, it is interesting to look at the dynamics in short length scales using the probe position correlation function $\Phi_{\mathbf{q}}^{\mathrm{s}}(t)$ for a large wave vector. Note that, as mentioned above, this correlation function is complex due to the symmetry breaking induced by the external force in the longitudinal direction, while it remains real in the transversal plane. Figure 5 presents the correlation functions for different forces up to the delocalization transition using a wave vector close to the location $q_{2}$ of the second peak in the structure factor of the host system $\left(q_{2} d \approx 12.75\right.$ in the theory and $q_{2} d \approx 13.35$ in the simulations). The panels in the top and middle rows of Fig. 5 show the real and imaginary parts for a wave vector parallel to the external force, respectively, and the bottom one for a wave vector perpendicular. Theory curves are plotted in the left column of panels, and simulation results in the right one, with qualitative agreement. Interestingly, the real part of the longitudinal correlation function can go below zero, given the symmetry breaking due to the force. The imaginary part, on the other hand, starts from zero and displays a maximum that moves to shorter times for increasing forces.

The theoretical correlation functions do not decay to zero irrespective of the wave vector direction, corresponding to a caged probe that cannot break free. The long-time plateau decreases upon increasing the force, indicating that the particle is less tightly localized. In the simulations, however, completely arrested probes are observed only for small forces (below $40 k_{\mathrm{B}} T / d$ ), while for moderate forces the correlation functions decay continuously, albeit slowly, to zero. This is in agreement with the nonarrested trajectories observed in Fig. 4 in the simulations.

The real part of the correlation function in the direction of the external force (top row of panels of the figure) shows a negative dip for moderate and large wave vectors in both the theory and the simulations. This negative dip shows the rattling of the probe in its cage of neighbors, enhanced by the external force, despite the microscopic dynamics being strongly damped (pure Brownian dynamics in the theory or Langevin in the simulations). Similar oscillations have been observed previously in microrheology in fluid states, with a similar theoretical model and corroborated by simulations [17]. In that case, the probe is delocalized for all forces, but the oscillations are more noticeable for strong pullings.

The wave-vector dependence of the correlation function is shown in Fig. 6 for the real and imaginary parts for wave vectors parallel to the external force from theory and simulations for a force deep in the localized regime. As observed in the figures, the wave-vector dependence in both components is rather involved. The real part of the correlation function (top row of panels of the figure) shows the typical microscopic relaxation to a finite long-time plateau (real part of the nonergodicity parameter, $f_{q}^{s}$ ), with a microscopic relaxation time that decreases with the wave vector, in both the theory and the simulations. Increasing the wave-vector modulus decreases the correlation function and becomes negative for long times and goes to zero for large wave vectors, as expected for short scales. The imaginary part shows the maximum mentioned above, which grows in intensity and moves to shorter times at moderate wave vectors. At long times, this component has a nonmonotonous behavior with the wave-vector modulus, with a maximum for wave vectors close to the first neighbor peak in the structure factor. These behaviors for both components are qualitatively reproduced by the simulations, as shown in the right column, although the long-time plateau is not reached for the smallest wave vectors studied. In particular, it must be noticed that the nontrivial evolution of the imaginary part is obtained.

Alternatively, the modulus and argument of this complex correlation function (wave vectors in the direction of the external force) can be studied, instead of the real and imaginary parts. The modulus of the correlation function decays monotonously with both time and wave vector to a long-time plateau. The phase starts from zero and grows to a long-time plateau and increases monotonously with the wave vector, reaching the maximum value $\pi$ for large wave vectors at long times.

The theoretical correlation functions for wave vectors in the plane perpendicular to the external force (bottom row) decay monotonously to a (positive) long-time plateau and decrease continuously for increasing wave vectors. These trends are correctly reproduced in the simulations, except for the small wave-vector modulus, where the long-time plateau is not reached. Overall, this component has the typical behavior of the host glass system, albeit with smaller long-time plateaus than the glass nonergodicity parameters.

The probe nonergodicity parameters (long-time plateaus of the probe position correlation functions) for different states in the localized regime are presented in Fig. 7, comparing again theory and simulations. Because the probe and bath particles are of the same size, the probe nonergodicity parameter gives the (real) glass bath nonergodicity parameter for vanishing forces. For finite forces, the longitudinal component becomes complex, and the real and imaginary parts are nonmonotonous, approaching $f_{q}^{s}=0$ for large $q$ (the real part from below and the imaginary part from above). If the modulus and argument of $f_{q}^{s}$ are studied, both of them have monotonous behaviors, $\left|f_{q}^{s}\right|$ decreases from 1 to 0 and arg $\left[f_{q}^{s}\right]$ increases from 0 to $\pi$. In any case, as the force increases, $f_{q}^{s}$ decays to zero faster, indicating that the probe is less and less 

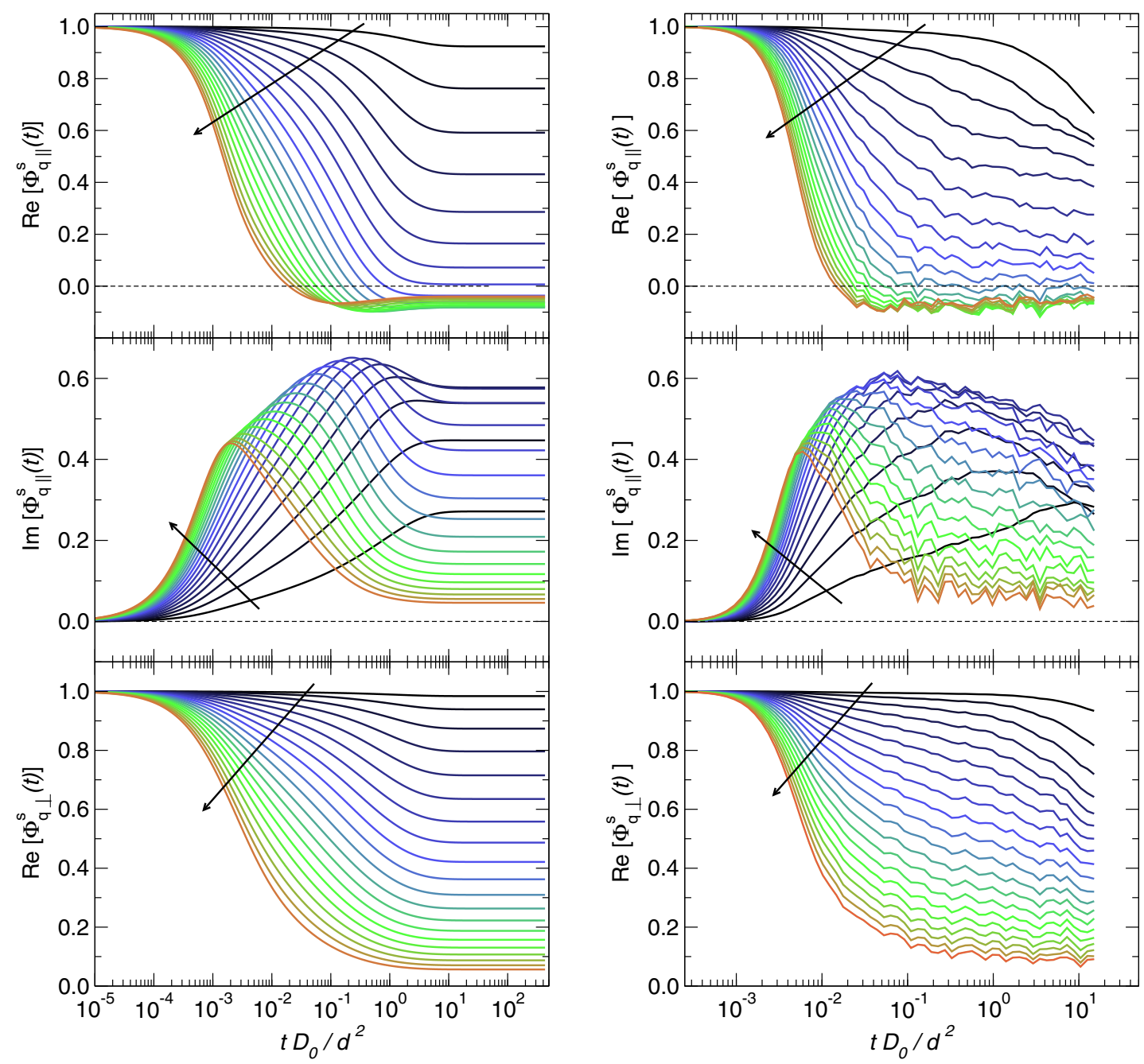

FIG. 6. Probe position correlation functions in the localized regime for $F=40 k_{\mathrm{B}} T / d$ and different wave vectors. The top and middle rows of panels show, respectively, real and imaginary parts of the correlation function in the direction of the force. Real-valued correlation functions perpendicular to the external force are shown in the bottom row. In the theory (left column of panels), $\phi=0.537$ and the wave vector ranges from $q d=1$ to $q d=20$. In the simulations (right column), $\phi=0.62$, the lines correspond to $q d$ values multiple of $2 \pi d / L=1.335$ ranging from $q d=1.335$ to $q d=26.5$. The arrows show the direction of increasing wave vector.

tightly caged, increasing its mobility, although it remains caged.

The measurement of the nonergodicity parameter in the simulations, on the other hand, is only possible for large wave vectors and not very large forces, where the long-time plateau is observed. Nevertheless, determining $f_{q}^{s}$ from the correlators at $t=5 \tau_{\mathrm{B}}$, the theoretical trends are fully confirmed by the simulations, in particular the change of sign in the real part of $f_{q \|}^{s}$ in the correct range of wave vectors, as well as the height and position of the maximum in its imaginary part. It must be mentioned that a quantitative comparison is hampered by the differences in the glass transition point of the bath $\left(\phi_{g}^{\mathrm{MCT}} \approx 0.5159\right.$ in the theory and $\phi_{g} \approx 0.596$ in the simulations) and critical forces.

\section{B. Shape of the cage of neighbors}

The probe nonergodicity parameter is the Fourier transform of the probe displacement distribution (or van Hove function of the probe) at long times, $G^{s}(\mathbf{r}, t \rightarrow \infty)$. In the localized regime, this gives the shape of the cage of neighbors as measured by the trapped probe. Figure 7 , therefore, can be more easily interpreted in real space. Figure 8 presents the distribution of probe displacements for different forces, comparing theory and simulations. For small forces, the cage retains its spherical symmetry, whereas for large forces, it deforms in the direction of the force. Additionally, the maximum of the distribution moves to positive values of $z$, even for very small forces, when the cage is not deformed, giving a measure of the diameter of the quiescent cage of neighbors: $0.1 d$ approximately. This value does not increase significantly for larger forces.

In the simulations, the distribution evolves with time, as already implied by the average probe displacement, Fig. 4, and does not reach a constant shape. Thus, we have calculated the distributions at a fixed time $\tau_{\mathrm{B}}=d^{2} / D_{0}$, but other values of the time give qualitatively the same results. The theoretical findings are confirmed. The maximum of the distribution does move to slightly larger values of $z$ upon increasing the external force, and the shape of the cage and the tails of the 

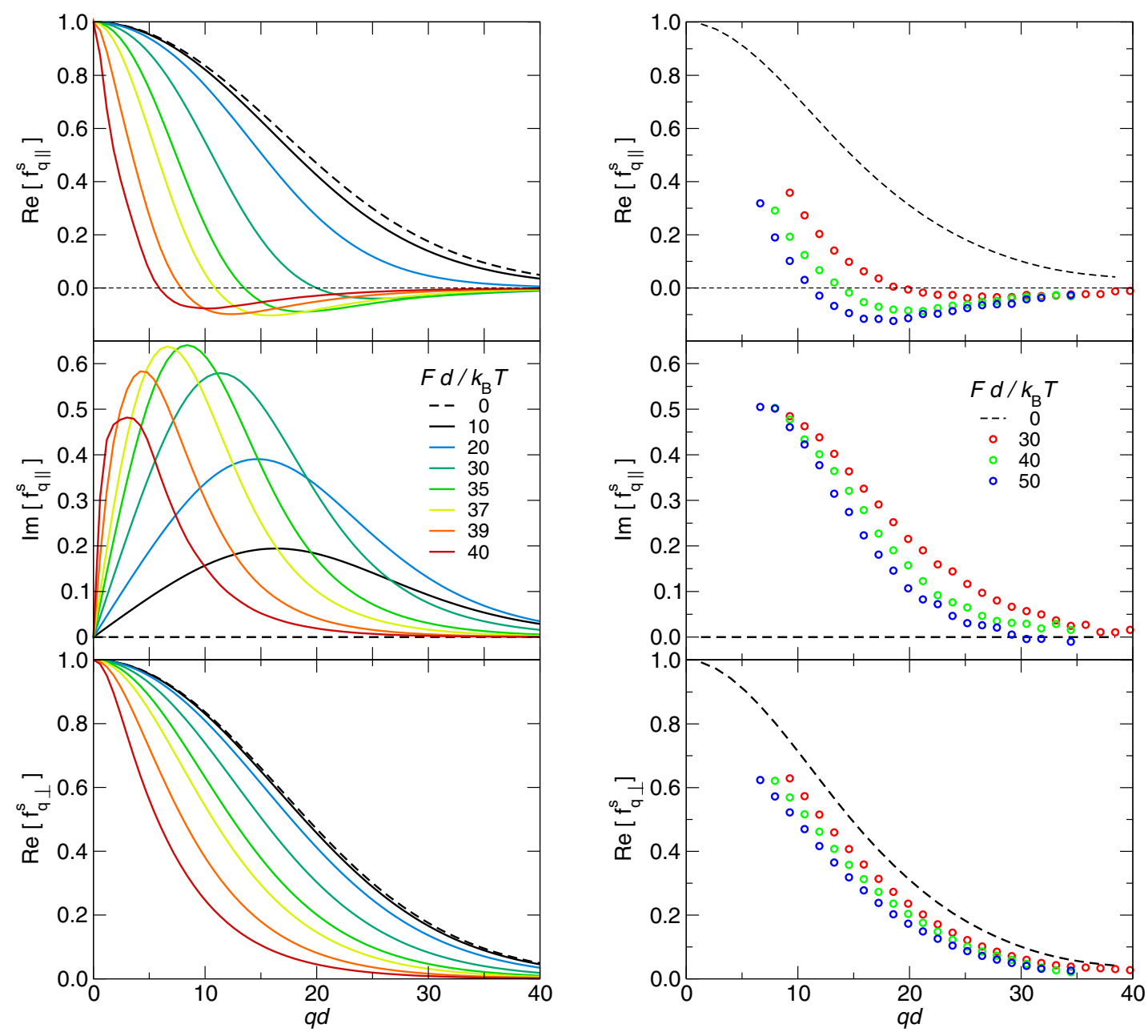

FIG. 7. Nonergodicity parameters as a function of wave-vector modulus for different forces in the localized regime. The top and middle rows of panels show the real and imaginary parts of $f_{q}^{s}$ with wave vectors in the direction of the external force, and the bottom row shows wave vectors in the perpendicular plane. In the theory (left column of panels), the host volume fraction is $\phi=0.537$ and the forces range from $F=0$ to $F=40 k_{\mathrm{B}} T / d$ as indicated. In the simulation (right column), $\phi=0.62$, the data correspond to forces $F=30,40$, and $50 k_{\mathrm{B}} T / d$ from top to bottom, and the black dashed line shows the force-free nonergodicity parameter.

distribution compare qualitatively well with the theory, despite the statistical inaccuracy in the simulations.

We have therefore studied integrated distributions in one direction: the longitudinal component $G_{\|}^{s}(z, t \rightarrow \infty)=$ $\int G^{s}(\mathbf{r}, t \rightarrow \infty) d \mathbf{r}_{\perp}$, where the integral is done over the transversal plane and $r_{\perp}$ stands for the transversal component of $\mathbf{r}$, and the transversal component $G_{\perp}^{s}\left(r_{\perp}, t \rightarrow \infty\right)=$ $\int G^{s}(\mathbf{r}, t \rightarrow \infty) d z$, with the integral along the direction of the force. Figure 9 presents both integrated components for different forces from theory and simulations. The longitudinal component (top row of panels), $G_{\|}^{s}(z, t \rightarrow \infty)$ ), is strongly asymmetric, with the maximum at small displacements, moving to longer displacements with increasing external forces, as expected from the plots in Fig. 8, and a tail extending to longer distances with increasing forces. In the transversal plane, $G_{\perp}^{s}\left(r_{\perp}, t \rightarrow \infty\right)$ shows a maximum at $r_{\perp}=0$ and nonGaussian tails to long distances. This reflects a heterogeneous dynamics of the probe inside the cage induced by the external force, capturing the intermittent dynamics observed in the simulations. These tails could be related to the exponential tails in the van Hove function observed in different supercooled systems [42] or models of activated dynamics [43], yet these were observed in structurally relaxing systems, while here we observe them in nonergodic states. Due to the external pulling, the probe has a small but finite probability of moving forward beyond the cage limits, although it cannot break free.

The distributions from the simulations are calculated at time $t=5 \tau_{\mathrm{B}}$, where we expect important differences between the maximum of the probe position distribution and $\langle\delta z\rangle$, from Fig. 4 (this time is also within the range where the probe correlator decays at $t^{-1 / 2}$ for the critical force, as shown below). The results, shown in the right column of panels of Fig. 9 present a maximum at small displacements and a slowly decaying tail to long distances, in agreement with the theory, also with the same behavior with respect to the external force. The inset in the top panel presents $G_{\perp}^{s}\left(r_{\perp}, t\right)$ at different times, which shows a slow evolution for times larger than $\approx 3 \tau_{\mathrm{B}}$. The width of the main peak at short distances remains constant, but the tail moves to longer distances, and a minimum in between develops. The average distributions, therefore, result from the presence of trajectories where the probe is confined within the cage of neighbors and other ones where the probe can escape 

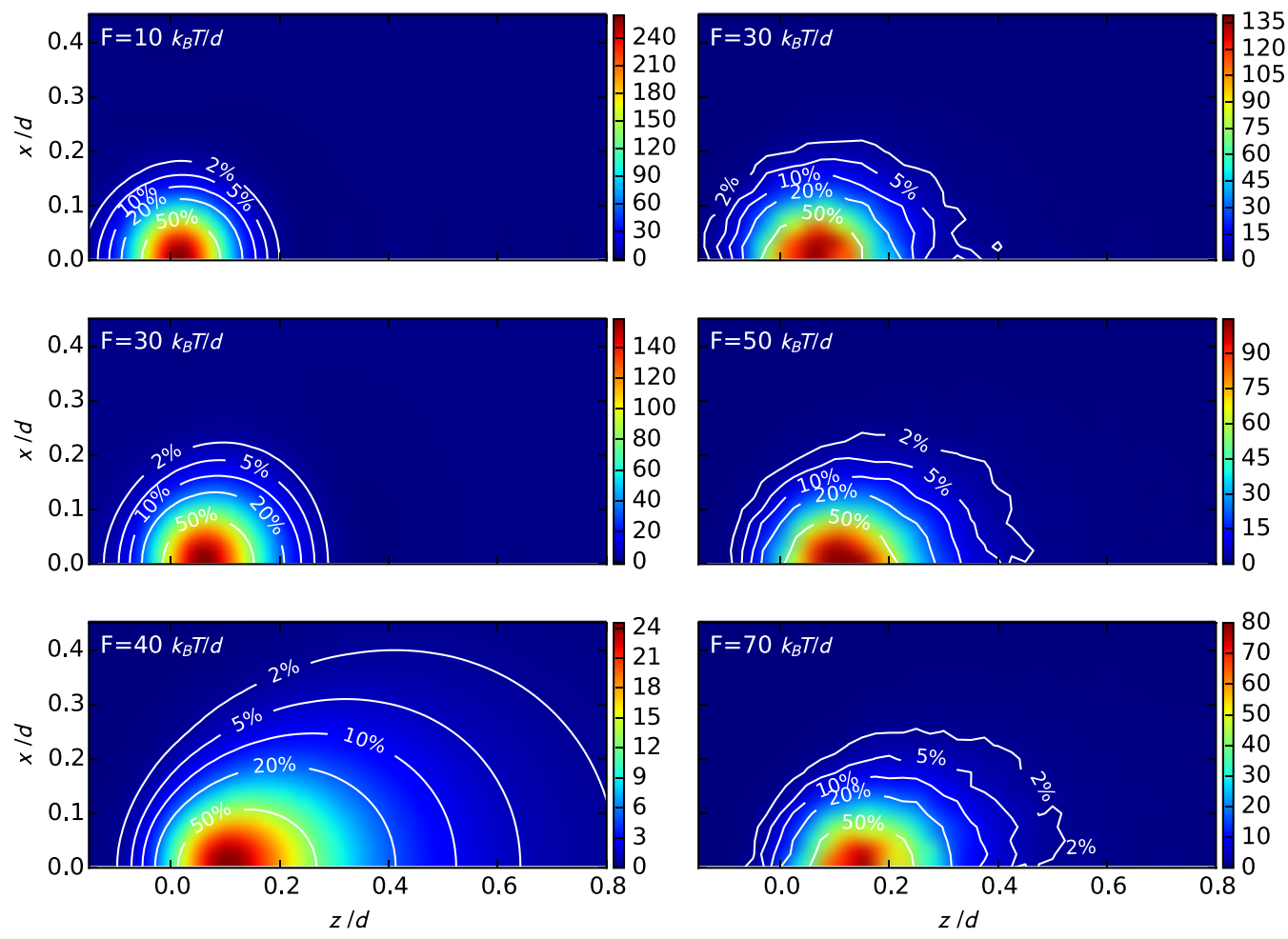

FIG. 8. Shape of the cage of neighbors explored by the probe for different forces increasing from top to bottom rows. In the theory (left column of panels) the data presents the distribution $G^{\mathrm{s}}(x, z ; t \rightarrow \infty)$ of probe displacements at long times (back Fourier transform of the probe nonergodicity parameter) for $F=10,30$, and $40 k_{\mathrm{B}} T / d$. In the simulations (right column) the probe displacement distribution at time $t=\tau_{\mathrm{B}}$ for $F=30,50$, and $70 k_{\mathrm{B}} T / d$ from top to bottom.

and travel to long distances (as shown in the inset to Fig. 4). As time passes, the fraction of particles that can escape and move to longer distances increases. In the range of forces studied here, however, this population does not disappear or move with a constant velocity even for the longer times studied.

In order to characterize the evolution of the distributions with the external force quantitatively, we study the position of the maximum of the distribution (indicative of the cage size, or localization length) and its first moment or average position (dominated by the tail of the distribution). Additionally, a typical length scale $\xi$ can be obtained by fitting $G_{\|}^{s} \propto \exp [-z / \xi]$ to the tails of the longitudinal distribution. Figure 10 shows these parameters as a function of the force, from the theoretical model (left panel) and simulations (right panel). Note that the decay length can be determined only in a narrow range of forces, where the tail is more significant, and follows the average distance. In the theory, while the position of the maximum grows slightly but keeps at short distances for all forces in the localized regime, the decay length grows dramatically as the critical force is approached. This is confirmed in the simulations, although the increase of the average position is extended over a wider range of forces than in the theory, probably because the these data are obtained at a finite time, $t=5 \tau_{\mathrm{B}}$. (Note the different range of forces studied in both cases.)

To further support the existence of a growing length scale as the critical force is approached, the second moment of the transversal distribution is also included in the graphs (the second moment is used because the dynamics in the perpendicular plane is diffusive, and not driven, as in the longitudinal direction). Up to a trivial scaling factor, the second moment follows the decay length of the longitudinal distribution, in both the theory and the simulations, indicating that a single growing correlation length appears in the system, dominated by the external force.

\section{Critical force}

The comparison between simulations and theory is made more difficult because the critical force in the simulated system is not yet known and the crossover appears broader in the simulations than numerically found in the theory. To strengthen the comparison, we seek a particular property of the critical force that can be used to establish unambiguously its existence in the simulations and locate it, even though the evolution on approaching it is smoother (see Fig. 4). Based on the similarity of our predictions with a pinning-depinning transition [44], a power-law decay of the probe position correlation function is expected. Note, however, that the mean-field model for the depinning transition focuses on the delocalized regime and provides little detail on the trapped probe below the threshold.

The dynamical critical law is found in the theoretical model with exponent $1 / 2, \Phi_{\mathbf{q}}^{\mathrm{s}}(t) \sim t^{-1 / 2}$, as shown in Fig. 11, where the correlation functions with wave vectors parallel and perpendicular to the force are presented. In the theory (left column), the power-law decay is observed only close to $F_{c}$, in a time range which increases the closer to it; $\epsilon_{F}=\left(F_{c}-F\right) / F_{c}$ gives the relative distance to the critical force. For forces 

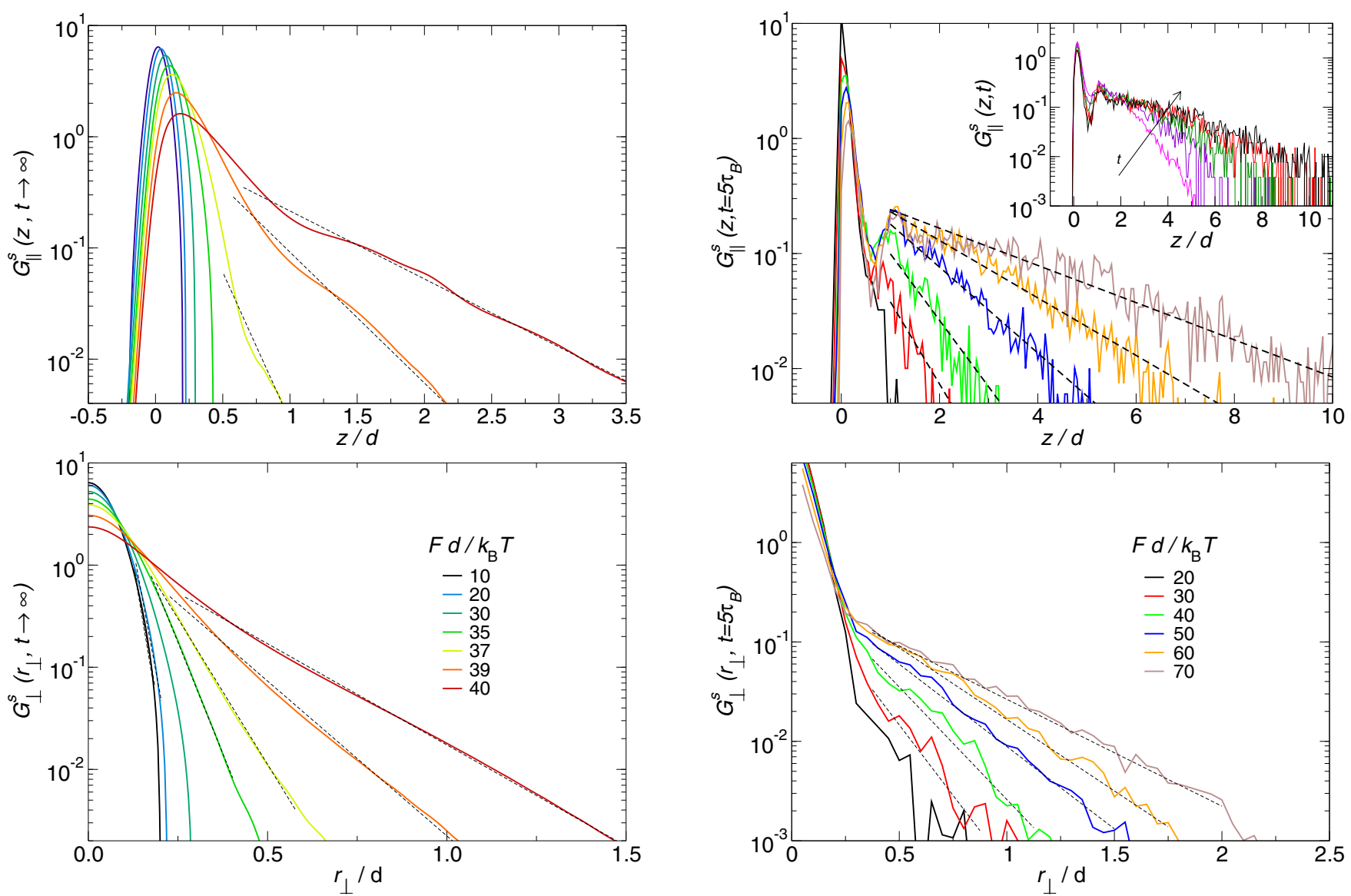

FIG. 9. Integrated components of the van Hove function for different forces: longitudinal components in the top row of panels and transversal ones in the bottom row (see text for the definition of the functions). The black dashed lines represent exponential fittings to the tails of the distributions at long distances. The inset shows the time evolution of the longitudinal component for $F=70 k_{\mathrm{B}} T / d$ and $t=\tau_{\mathrm{B}}, 2 \tau_{\mathrm{B}}, 3 \tau_{\mathrm{B}}, 4 \tau_{\mathrm{B}}$, and $5 \tau_{\mathrm{B}}$ (increasing from bottom to top).

smaller than $F_{c}$ the correlation function levels off to a plateau that decreases with the force, while for larger forces it decays faster to zero. The derivation of the critical $t^{-1 / 2}$ law by a $\beta$-scaling analysis [6] of our fully $q$-dependent MCT equations (and also of a reduced schematic model) will be published separately.

In the simulations, the gap between the forces studied is too large to produce a similar picture, but the power-law decay
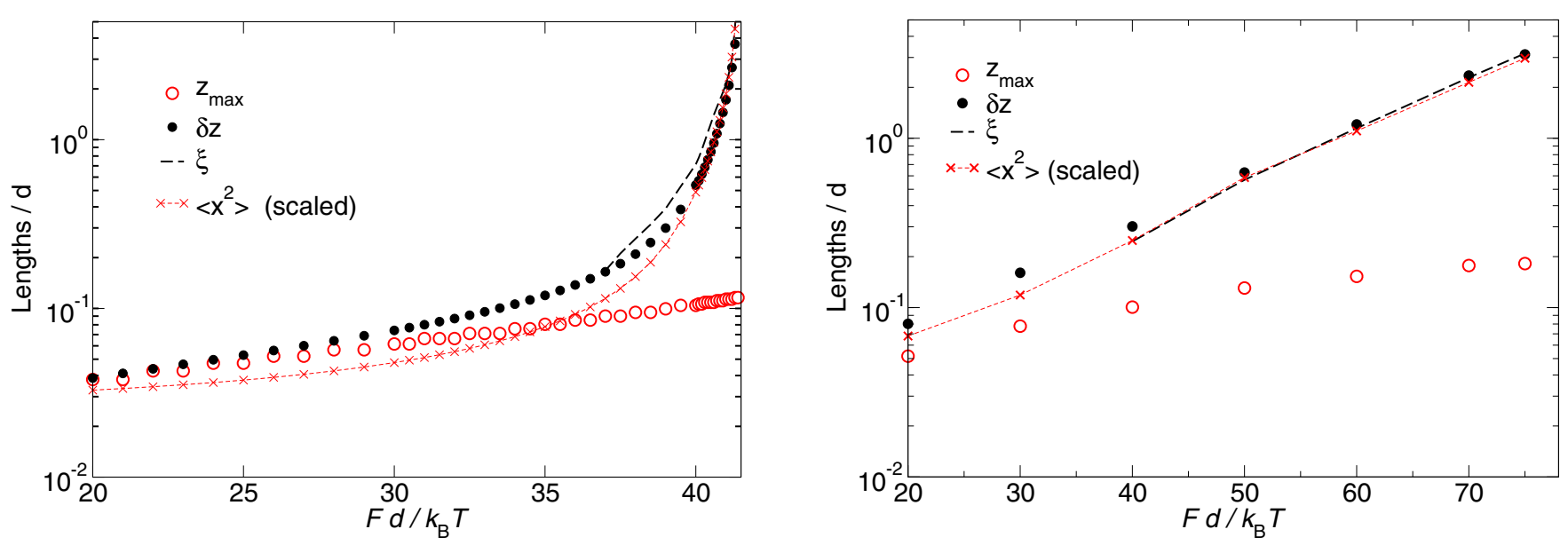

FIG. 10. Maximum and first moment of the longitudinal distribution as a function of the external force from theory (left) and simulations (right). The dashed lines present the correlation length $\xi$ obtained from exponential fittings $G_{\|}^{s} \propto \exp [-z / \xi]$ to the tails of the distributions. The (scaled) second moment of the distribution in the perpendicular plane is also plotted in both cases. 

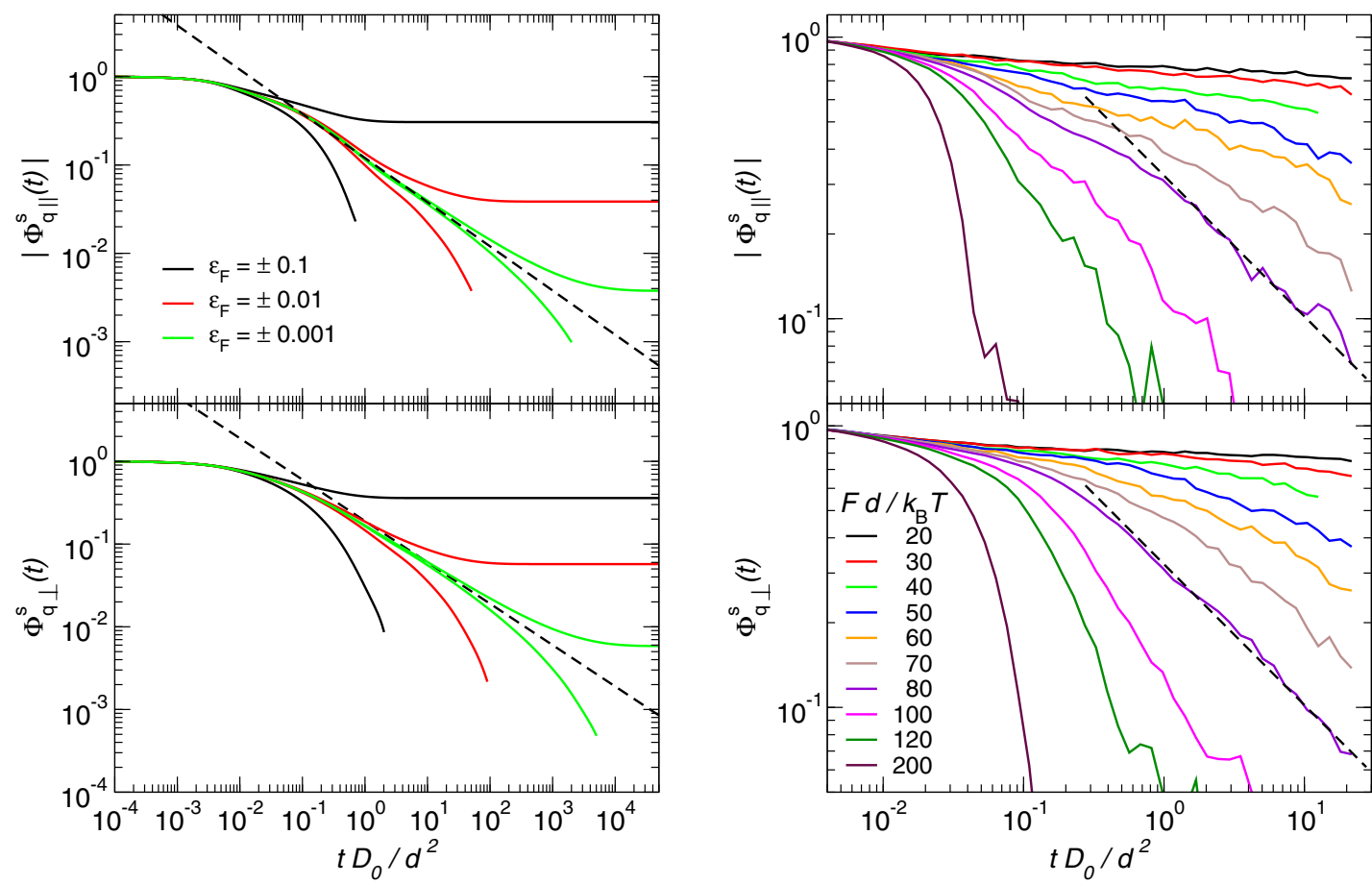

FIG. 11. Probe position correlation functions in log-log scale for a wave vector close to the location $q_{1}$ of the first neighbor peak in the structure factor $\left(q_{1} d \approx 7\right.$ in the theory and $q_{1} d \approx 7.95$ in the simulations), parallel (top row of panels) and perpendicular to the external force. In the parallel direction the modulus of the function is presented. Theory (left column of panels) and simulations (right column) are compared. The dashed line shows the $t^{-1 / 2}$ behavior in all panels.

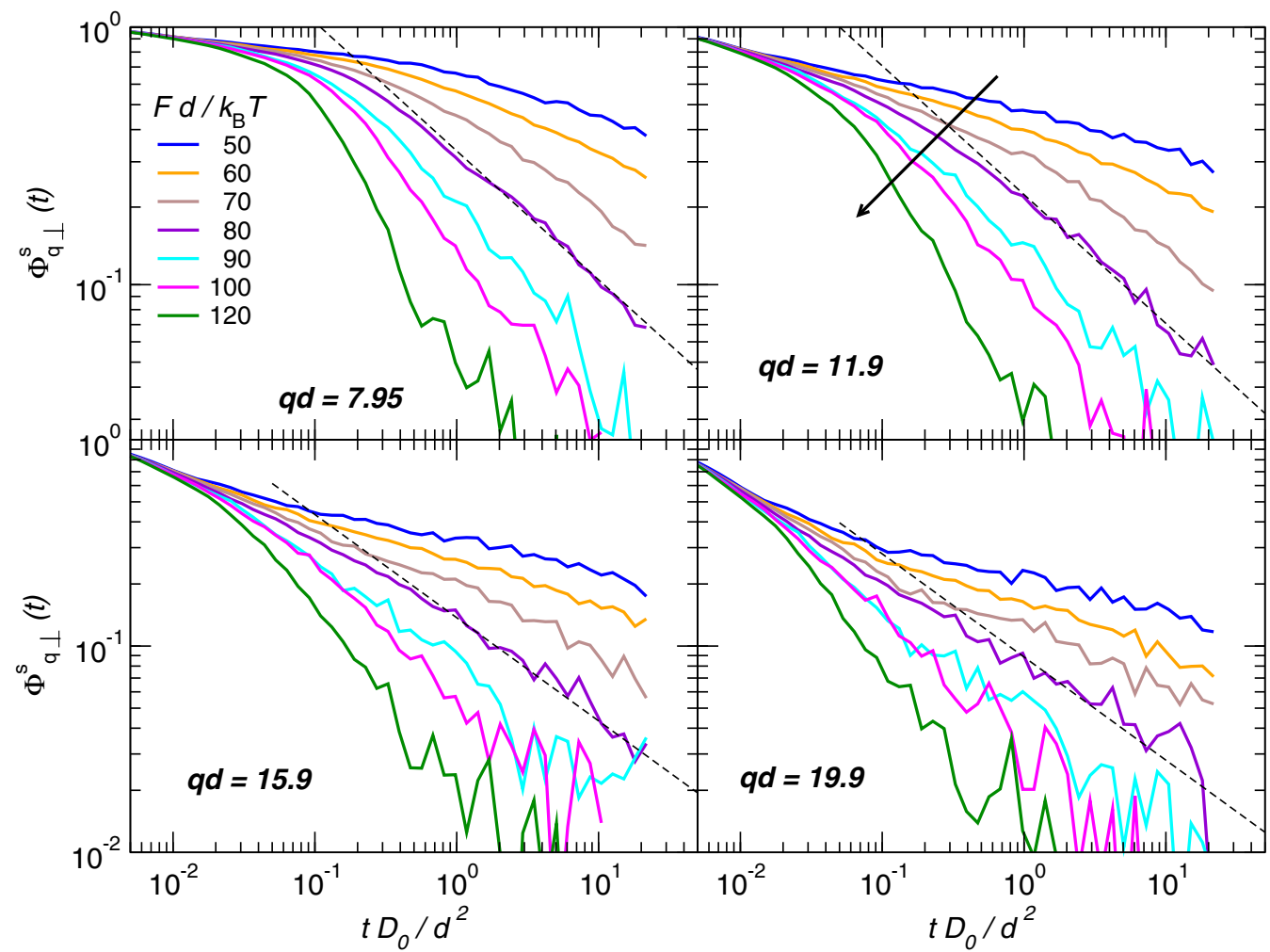

FIG. 12. Probe position correlation functions for different wave vectors perpendicular to the external force, as labeled, from simulations. The dashed lines show the $t^{-1 / 2}$. 
is indeed observed for $F=80 k_{\mathrm{B}} T / d$, for almost two decades in time, in both directions of the wave vector. Interestingly, this force corresponds in Fig. 4 to the value where the longtime probe displacement crosses over from sublinear to linear. It must be noted, however, that the correlation functions for smaller forces decrease continuously in the time range studied and do not show any indication of leveling off, as predicted by the theory.

To further support observation of the power-law decay of the correlation function in the simulations, with the predicted behavior, in Fig. 12 the correlation functions for different wave vectors perpendicular to the external force are presented. For all wave vectors, the power-law decay is found in the same time range, whereas all other forces deviate from this behavior. In particular, the correlation function for $F=70 k_{\mathrm{B}} T / d$ that apparently follows the $t^{-1 / 2}$ behavior for low wave vectors deviates clearly for large ones.

The value of the critical force can be used to estimate the elasticity of the cage of neighbors, as measured by the probe. Mode-coupling theory gives for the spring constant of the cage (in the linear-response approximation) a value of $\approx 200 k_{\mathrm{B}} T / d^{2}$ at the critical packing fraction. This compares quite well with simulations of passive microrheology in the same system in undercooled conditions, volume fraction $\phi_{c} \sim 0.58$, that yielded an estimate of the cage strength of ca. $320 k_{\mathrm{B}} T / d^{2}$ [45]. These values give restoring forces of $\sim 40 k_{\mathrm{B}} T / d$ in the theory and $\sim 65 k_{\mathrm{B}} T / d$ in the simulations (using a cage size of $0.2 d$ ). Our results from active microrheology for the critical force, $F_{c}=$ $46 k_{\mathrm{B}} T / d$ in the theory and $F_{c} \sim 80 k_{\mathrm{B}} T / d$ in the simulations, agree quite well with this value, considering that the present system is denser, and therefore the cage should be stronger.

\section{CONCLUSIONS}

We have investigated theoretically and by simulations the dynamics of a probe driven by a constant force through a colloidal glass of hard spheres. Our extended, fully microscopic mode-coupling theory predicts an ideal localized state of the probe and a sharp delocalization transition with a well-defined critical force. Simulations show a broader crossover, being the critical force defined with the help of the theoretically predicted critical power-law decay of the probe correlation function.

The present theory qualitatively reproduces the main features of the probe dynamics on the local length scale (the decay of the probe correlation functions and the deformation of the cage) observed in simulations. Both theory and simulations show growing correlation lengths (arising in the exponential tails of the probe van Hove function) as the force on the probe approaches the critical value. Exponential tails quantify dynamical heterogeneities, which manifest themselves in the intermittent motion of the probe observed in the simulated probe trajectories.

We showed that the extended MCT formulation recovers the cylindrical symmetry present in the microrheology setup and provides (except for small errors known from forcefree cases [37]) positive real-space van Hove functions. It is also capable of describing the probe dynamics in a fluid host and under large forces in the delocalized regime. It allows for a theoretical investigation of the generalized
Stokes-Einstein relation (GSER) [46,47], which connects the frequency-dependent probe friction coefficient (obtained from the transient drift velocity) to the macroscopic rheology. This will be the subject of a future publication.

\section{ACKNOWLEDGMENTS}

We are indebted to Thomas Voigtmann for helpful hints and discussions. Financial support from the Deutsche Forschungsgemeinschaft in Project No. P3 of Grant No. FOR1394 is acknowledged. A.M.P. is financially supported by the Spanish Ministerio de Economía y Competitividad and FEDER under Project No. FIS2015-69022P.

\section{APPENDIX A: CYLINDRICAL SYMMETRY OF THE FULL PROBLEM}

We want to show that the correlators are cylindrically symmetric under the assumption that the potential $U$ from which the interparticle forces $\mathbf{F}_{j}$ are derived is isotropic; i.e., it depends only on the distance between the particles, $U\left(Q \mathbf{r}_{1}, \ldots, Q \mathbf{r}_{N}\right)=U\left(\mathbf{r}_{1}, \ldots, \mathbf{r}_{N}\right)$. As already pointed out in Sec. II C it is equivalent to show the cylindrical symmetry of the correlator to prove relation (34). We start with writing the fundamental definition (28) as an explicit integral:

$$
\begin{aligned}
m_{Q \mathbf{q}}^{\alpha \beta}(t)= & \int d X \Psi_{\mathrm{eq}}(X) Q_{s}\left(\mathbf{r}_{1}\right) F_{1}^{\alpha}(X) e^{-i Q \mathbf{q} \cdot \mathbf{r}_{1}} \\
& \times Q_{s}\left(\mathbf{r}_{1}\right) e^{\Omega_{\mathrm{irr}}^{\dagger}(X) t} F_{1}^{\beta}(X) e^{i Q \mathbf{q} \cdot \mathbf{r}_{1}} .
\end{aligned}
$$

Since $Q \mathbf{q} \cdot \mathbf{r}_{1}=\mathbf{q} \cdot Q^{T} \mathbf{r}_{1}$, the idea is to do a substitution $\mathbf{r}_{j}^{\prime}=Q^{T} \mathbf{r}_{j}$ for $j=1, \ldots, N$. As the Jacobian of this transformation has determinant 1 , it remains to calculate how the objects in the integral transform under this substitution. Direct calculation shows $Q_{1}\left(\mathbf{r}_{1}\right)=Q_{1}\left(\mathbf{r}_{1}^{\prime}\right)$, since we sum over all wave vectors. The chain rule for differentiation implies $\boldsymbol{\partial}_{j}=Q \boldsymbol{\partial}_{j}^{\prime}$ and, in particular, $\mathbf{F}_{j}(X)=Q \mathbf{F}_{j}\left(Q X^{\prime}\right)=Q \mathbf{F}_{j}\left(X^{\prime}\right)$ by the assumption of an isotropic potential. Using these results, we find $\Omega_{\mathrm{eq}}^{\dagger}(X)=\Omega_{\mathrm{eq}}^{\dagger}\left(X^{\prime}\right)$, because $Q$ is an isometry. The relation $\Delta \Omega^{\dagger}(X)=\Delta \Omega^{\dagger}\left(X^{\prime}\right)$ is only valid because we required $Q \hat{\mathbf{z}}=\hat{\mathbf{z}}$. Here one can see explicitly that the system is not isotropic but only cylindrically symmetric. Again using the transformation rules, one finds that the irreducible operator is invariant, too. Hence, we conclude $\exp \left[\Omega_{\text {irr }}^{\dagger}(X)\right]=$ $\exp \left[\Omega_{\text {irr }}^{\dagger}\left(X^{\prime}\right)\right]$. Finally, we have $\Psi_{\text {eq }}(X)=\Psi_{\text {eq }}\left(X^{\prime}\right)$ due to the isotropic potential. Thus, we can identify $m_{\mathbf{q}}^{\gamma \delta}(t)$ after the substitution and obtain

$$
m_{Q \mathbf{q}}^{\alpha \beta}(t)=\sum_{\gamma, \delta} Q^{\alpha \gamma} m_{\mathbf{q}}^{\gamma \delta}(t)\left(Q^{T}\right)^{\delta \beta},
$$

which was the claim.

\section{APPENDIX B: AXISYMMETRIC MCT KERNEL}

Under the assumption of axial symmetry the MCT form (52) for the friction kernel $\mathbf{m}_{\mathbf{q}}(t)$ must be invariant under rotations in the direction $\hat{\mathbf{z}}$ of the external force. This condition yields

$$
\mathbf{m}_{\mathbf{q}}(t)=\int_{-\infty}^{\infty} d k_{z} \int_{0}^{\infty} d k_{x} k_{x} \mathbf{J}(\mathbf{q}, \mathbf{k} ; t) \Phi^{\mathrm{s}}(\mathbf{k} ; t),
$$


where the tensor $\mathbf{J}$ contains the MCT vertex and the host correlator. Explicit expressions for the relevant elements of $\mathbf{J}$ are given below [31]:

$$
\begin{aligned}
J^{x x}(\mathbf{q}, \mathbf{k} ; t)= & \left(\frac{k_{x}}{2}+q_{x}^{2}\right) \bar{g}_{0}(\mathbf{q}, \mathbf{k} ; t)-2 q_{x} k_{x} \bar{g}_{1}(\mathbf{q}, \mathbf{k} ; t) \\
& +\frac{k_{x}^{2}}{2} \bar{g}_{2}(\mathbf{q}, \mathbf{k} ; t),
\end{aligned}
$$

$$
\begin{aligned}
& J^{z z}(\mathbf{q}, \mathbf{k} ; t)=\left(q_{z}-k_{z}\right)^{2} \bar{g}_{0}(\mathbf{q}, \mathbf{k} ; t), \\
& J^{x z}(\mathbf{q}, \mathbf{k} ; t)=\left(q_{z}-k_{z}\right)\left[q_{x} \bar{g}_{0}(\mathbf{q}, \mathbf{k} ; t)-k_{x} \bar{g}_{1}(\mathbf{q}, \mathbf{k} ; t)\right],
\end{aligned}
$$

where

$$
\bar{g}_{i}(\mathbf{q}, \mathbf{k} ; t)=\int_{0}^{2 \pi} d \theta \cos (i \theta) g[p(\mathbf{q}, \mathbf{k}, \cos \theta) ; t],
$$

for $i=0,1,2$,

$p(\mathbf{q}, \mathbf{k}, \cos \theta)=\sqrt{q_{x}^{2}+k_{x}^{2}+\left(q_{z}-k_{z}\right)^{2}-2 q_{x} k_{x} \cos (\theta)}$,

and

$$
g(p ; t)=\frac{1}{(2 \pi)^{3}} \frac{\left(S_{p}^{\mathrm{s}}\right)^{2}}{n S_{p}} \Phi_{p}(t) .
$$

For actual numerical calculations, the integral (B1) is approximated by the quadrature

$$
\begin{aligned}
\mathbf{m}_{\mathbf{q}}(t) \approx & \sum_{i=0}^{N_{x}} \sum_{j=0}^{N_{z}} k_{x}(i) w_{x}(i) w_{z}(j) \\
& \times \mathbf{J}\left[\mathbf{q}, k_{x}(i), k_{z}(j) ; t\right] \Phi^{\mathrm{s}}\left[k_{x}(i), k_{z}(j) ; t\right],
\end{aligned}
$$

where $w_{x}$ and $w_{z}$ are the quadrature weights in perpendicular and parallel direction, respectively.

The wave vectors $\mathbf{q}$ and $\mathbf{k}$ take discrete values on a twodimensional grid $\left(q_{x}, q_{z}\right)$, with $0 \leqslant q_{x} \leqslant Q$ and $-Q \leqslant q_{z} \leqslant Q$, $Q$ being the wave-number cutoff. A starting grid is formed by $N \times 2 N$ points equidistantly distributed in each direction with spacing $\Delta$.

As the critical force $F_{c}$ on the probe is approached, the nonergodicity parameter $f_{q}^{s}$ develops a sharp peak around $q=0$. This trend can be seen on the left panel of Fig. 7 with increasing force. Therefore, the theoretical value of $F_{c}$ (calculated using a static fixed point iteration [Eq. (54)] as described at the end of Sec. IIE) depends on how this peak is resolved in our $\mathbf{q}$ grid of wave vectors.

To capture the features of the probe correlator and memory functions at small wave vectors and $F \lesssim F_{c}$, we add to the regular starting grid $\bar{N} \times 2 N$ more points $\left(q_{x}, q_{z}\right)$ with $0<$ $q_{x}<\Delta$ and $N \times 2 \bar{N}$ more points $\left(q_{x}, q_{z}\right)$ with $-\Delta<q_{z}<\Delta$. These additional points are spaced in a way that the grid is logarithmically refined towards the origin $\mathbf{q}=0$ along each direction.

In the present calculations, we have used $\bar{N}=10$. For given $N$ and cutoff $Q$, further increase of $\bar{N}$ produces no significant changes (less than $1 \%$ ) on the critical force $F_{c}$. Calculation of the probe dynamics by solving Eq. (4) at a given force $F$ uses the same q grid used for solving Eq. (54) in static calculations. In this way the relative separation $\varepsilon_{F}=\left(F_{c}-F\right) / F_{c}$ to the critical force is consistently defined.
The static calculations shown use a cutoff of $q d=50$ in order to get fewer artifacts in the inverse Fourier transform. As the dynamic calculations are computationally more expensive, a cutoff of $q d=10$ is used, which shifts the critical force from $41.6 k_{\mathrm{B}} T / d$ for the static calculations to $46.0 k_{\mathrm{B}} T / d$ for the dynamic calculations.

\section{APPENDIX C: NUMERICAL SOLUTION FOR THE PROBE CORRELATOR}

In this section we briefly sketch the procedure to numerically solve the memory equation (4) for the probe correlator $\Phi_{\mathbf{q}}^{\mathrm{s}}(t)$.

We consider a decimated time grid [48],

$$
t=t_{i}^{(d)}=i h 2^{d}, \quad i=0, \ldots, n, \quad d=0,1, \ldots, D,
$$

where $h$ is the fundamental time step, $d$ indexes the decimation level, $n$ the number of time points at each level, and $D$ the total number of decimations. The total evolution time is $T=n 2^{D} h$. Henceforth, in the text we use the abbreviated notation $t_{i}^{(d)} \equiv i$ for conciseness.

First the values of $\Phi_{\mathbf{q}}^{\mathrm{s}}(i)$ are initialized in the short time grid, corresponding to $d=0$. This is done by using an approximate short-time solution of Eq. (4), which can be obtained by neglecting the memory integral in (4).

To calculate $\Phi_{\mathbf{q}}^{\mathrm{s}}(i)$ at later times, we consider coarser decimation levels $d \geqslant 1$. We first map the set of $n$ already known values $\Phi_{\mathbf{q}}^{\mathrm{s}}(i)$ at the level $d-1$ into the first $n / 2$ points of the time grid at level $d$,

$$
\Phi_{\mathbf{q}}^{\mathrm{s}}(i)=\Phi_{\mathbf{q}}^{\mathrm{s}}(2 i), \quad i=1, \ldots, n / 2 .
$$

This procedure is called decimation.

The unknown values of $\Phi_{\mathbf{q}}^{\mathrm{s}}(i)$ at the remaining points $i=n / 2+1, \ldots, n$ of the grid at level $d$ are calculated by a fixed-point iteration of the form

$$
\left[\Phi_{\mathbf{q}}^{\mathrm{s}}(i)\right]^{(k+1)}=\mathrm{F}_{\mathbf{q}}\left\{\left[\boldsymbol{\Phi}^{\mathrm{s}}(i)\right]^{(k)} ; \boldsymbol{\Phi}^{\mathrm{s}}(j<i)\right\},
$$

for $k=0,1,2, \ldots$ An expression for the iteration functional $F_{q}$ is obtained from a proper discretized form of the memory equation (4) on the time grid at level $d$ (see, e.g., Ref. [48]). For each $i$ the sequence of iterates in (C3) may be initialized by setting $\left[\Phi_{\mathbf{q}}^{\mathrm{s}}(i)\right]^{(0)}=\Phi_{\mathbf{q}}^{\mathrm{s}}(i-1)$.

The functional form (C3) means that $\Phi_{\mathbf{q}}^{\mathrm{s}}(i)$ depends on all $\Phi_{\mathbf{q}}^{\mathrm{s}}(j)$ at previous times $(j<i)$ and also (implicitly) on $\Phi_{\mathbf{q}}^{\mathrm{s}}(i)$ at the same time $i$ through the memory function $m_{\mathbf{q}}(i)$. Each iteration step $(k+1)$ in $(\mathrm{C} 3)$ requires the evaluation of the effective memory $\left[m_{\mathbf{q}}(i)\right]^{(k)}$. For the present case of multiple relaxation channels, $m_{\mathbf{q}}(i)$ exhibits a nonlocal (in time) dependence on the probe correlators $\Phi_{\mathbf{q}}^{\mathrm{s}}$. This may be written formally in functional form as

$$
\left[m_{\mathbf{q}}(i)\right]^{(k)}=\mathrm{G}_{\mathbf{q}}\left\{\left[\boldsymbol{\Phi}^{\mathrm{s}}(i)\right]^{(k)} ; \boldsymbol{\Phi}^{\mathrm{s}}(j<i)\right\} .
$$

An explicit expression for the functional $\mathbf{G}_{\mathbf{q}}$ (to be derived later in Sec. V) is obtained by discretizing the integral equation (41) on the decimated time grid.

The procedure described above exploits the causality property of the memory equation (4); that is, $\Phi_{\mathbf{q}}^{\mathrm{s}}(t)$ are determined by the correlators $\Phi_{\mathbf{q}}^{\mathrm{s}}\left(t^{\prime}\right)$ for $t^{\prime}$ preceding $t$. Causality is preserved by the MCT approximation for the 
effective functional (5), as can be seen from Eqs. (52) and (41), although the effective memory $m_{\mathbf{q}}(t)$ is, in fact, nonlocal in time.

An explicit expression for the functional (C3) for direct numerical implementation can obtained by a well-established procedure [48] and is given below:

$$
\Phi_{\mathbf{q}}^{\mathrm{s}}(i)=\frac{\beta}{\alpha} m_{\mathbf{q}}(i)-\frac{\gamma}{\alpha},
$$

where

$$
\begin{aligned}
\alpha= & \frac{3}{2} \frac{1}{2^{d} h}+\Gamma_{\mathbf{q}}+d m_{\mathbf{q}}(1), \\
\beta= & \Phi_{\mathbf{q}}^{\mathrm{s}}(0)-d \Phi_{\mathbf{q}}^{\mathrm{s}}(1), \\
\gamma= & \frac{1}{2^{d} h}\left[\frac{1}{2} \Phi_{\mathbf{q}}^{\mathrm{s}}(i-2)-2 \Phi_{\mathbf{q}}^{\mathrm{s}}(i-1)\right] \\
& +m_{\mathbf{q}}(i-\bar{l}) \Phi_{\mathbf{q}}^{\mathrm{s}}(\bar{l})+I_{1}^{\prime}+I_{2}^{\prime},
\end{aligned}
$$

with

$$
\begin{aligned}
I_{1}^{\prime}= & -m_{\mathbf{q}}(i-1) d \Phi_{\mathbf{q}}^{\mathrm{s}}(1) \\
& +\sum_{k=2}^{\bar{\imath}}\left[m_{\mathbf{q}}(i-k+1)-m_{\mathbf{q}}(i-k)\right] d \Phi_{\mathbf{q}}^{\mathrm{s}}(k) \\
I_{2}^{\prime}=- & \Phi_{\mathbf{q}}^{\mathrm{s}}(i-1) d m_{\mathbf{q}}(1) \\
& +\sum_{k=2}^{i-\bar{l}}\left[\Phi_{\mathbf{q}}^{\mathrm{s}}(i-k+1)-\Phi_{\mathbf{q}}^{\mathrm{s}}(i-k)\right] d m_{\mathbf{q}}(k),
\end{aligned}
$$

and moments

$$
d \Phi_{\mathbf{q}}^{\mathrm{s}}(k) \equiv \frac{1}{2^{d} h} \int_{t_{k-1}}^{t_{k}} \Phi_{\mathbf{q}}^{\mathrm{s}}\left(t^{\prime}\right) d t^{\prime},
$$

for $k=1, \ldots, i-\bar{\imath}$, and the same for $d m_{\mathbf{q}}(k)$.

At the finest decimation level $(d=0)$, the moments are approximated by the trapezoidal rule

$$
d \Phi_{\mathbf{q}}^{\mathrm{s}}(k) \approx\left[\Phi_{\mathbf{q}}^{\mathrm{s}}(k-1)+\Phi_{\mathbf{q}}^{\mathrm{s}}(k)\right] / 2,
$$

for $k=1, \ldots, n / 2$. For $d \geqslant 1$ the moments are decimated as

$$
d \Phi_{\mathbf{q}}^{\mathrm{s}}(k) \approx\left[d \Phi_{\mathbf{q}}^{\mathrm{s}}(2 k-1)+d \Phi_{\mathbf{q}}^{\mathrm{s}}(2 k)\right] / 2,
$$

for $k=1, \ldots, n / 4$, and

$$
d \Phi_{\mathbf{q}}^{\mathrm{s}}(k) \approx\left[\Phi_{\mathbf{q}}^{\mathrm{s}}(2 k-2)+2 \Phi_{\mathbf{q}}^{\mathrm{s}}(2 k-1)+\Phi_{\mathbf{q}}^{\mathrm{s}}(2 k)\right] / 4,
$$

for $k=n / 4+1, \ldots, n / 2$. The same expressions are used to approximate $d m_{\mathbf{q}}(k)$.

We take $\bar{\imath}=\lfloor i / 2\rfloor$, where $\lfloor x\rfloor$ gives the largest integer less than or equal to $x$ (the floor function).

\section{APPENDIX D: NUMERICAL CALCULATION OF THE EFFECTIVE MEMORY KERNEL}

Here we derive an explicit expression for the functional relation (C4). For conciseness we note that, for each wave number, the integral equation (41) for the effective memory kernel has the form of a Volterra integral equation [49],

$$
X(t)+\int_{0}^{t} A\left(t-t^{\prime}\right) X\left(t^{\prime}\right) d t^{\prime}=B(t),
$$

where $A(t)$ and $B(t)$ are known functions and $X(t)$ is to be determined. Here $X(t)$ stands for $m_{\mathbf{q}}(t)$ and $A(t)$ for $\alpha_{\mathbf{q}}(t)$ in (41), while $B(t)$ denotes the entire right-hand side of Eq. (41).

First, Eq. (D1) is solved in the short-time grid, corresponding to $d=0$ in (C1). We approximate the integral in (D1) by a numerical quadrature,

$$
X(i)+\sum_{j=0}^{i} w_{i j} A(i-j) X(j)=B(j),
$$

for $i=0, \ldots, n$, where $w_{i j}$ are the quadrature weights. Equation (D2) can be explicitly solved for $X(i)$,

$$
X(i)=\frac{1}{1+w_{i i} A(0)}\left[B(i)-\sum_{j=0}^{i-1} w_{i j} A(i-j) X(j)\right]
$$

for $i=1, \ldots, n$, with $X(0)=B(0)$.

To calculate $X(i)$ at later times, we consider coarser decimation levels $d \geqslant 1$. It starts from the decimation

$$
X(i)=X(2 i), \quad i=0, \ldots, n / 2,
$$

and the same for $A(i)$ and $B(i)$.

The unknown values of $X(i)$ at the remaining points $i=n / 2+1, \ldots, n$ of the grid at level $d$ are calculated from a discretized form of the integro-differential equation

$$
\dot{X}(t)+\frac{d}{d t} \int_{0}^{t} A\left(t-t^{\prime}\right) X\left(t^{\prime}\right) d t^{\prime}=\dot{B}(t),
$$

with initial condition $X(0)=B(0)$. Equation (D5) was derived from (D1) by differentiation with respect to $t$. Next we discretize Eq. (D5) in the decimated time grid.

Using Leibniz integral rule, integration by parts, and a change of variables, one may show that

$$
\begin{aligned}
& \frac{d}{d t} \int_{0}^{t} A\left(t-t^{\prime}\right) X\left(t^{\prime}\right) d t^{\prime}=\int_{0}^{\bar{t}} \dot{A}\left(t-t^{\prime}\right) X\left(t^{\prime}\right) d t^{\prime} \\
& \quad+\int_{0}^{t-\bar{t}} \dot{X}\left(t-t^{\prime}\right) A\left(t^{\prime}\right) d t^{\prime}+A(t-\bar{t}) X(\bar{t}),
\end{aligned}
$$

for $0<\bar{t}<t$.

We take $t=i 2^{d} h$ and $\bar{t}=\bar{\imath} 2^{d} h$ in the time grid at level $d$ and approximate the first integral in the right-hand side of Eq. (D6) by

$$
\begin{gathered}
\int_{0}^{\bar{t}} \dot{A}\left(t-t^{\prime}\right) X\left(t^{\prime}\right) d t^{\prime}=\sum_{k=1}^{\bar{\imath}} \int_{t_{k-1}}^{t_{k}} \dot{A}\left(t_{i}-t^{\prime}\right) X\left(t^{\prime}\right) d t^{\prime} \\
\quad \approx \sum_{k=1}^{\bar{\imath}}\left[\frac{A\left(t_{i}-t_{k-1}\right)-A\left(t_{i}-t_{k}\right)}{2^{d} h}\right] \int_{t_{k-1}}^{t_{k}} X\left(t^{\prime}\right) d t^{\prime} \\
=\sum_{k=1}^{\bar{\imath}}[A(i-k+1)-A(i-k)] d X(k) \equiv I_{1},
\end{gathered}
$$

where we have defined the moments

$$
d X(k) \equiv \frac{1}{2^{d} h} \int_{t_{k-1}}^{t_{k}} X\left(t^{\prime}\right) d t^{\prime}, \quad k=1, \ldots, \bar{l} .
$$


Using the same route for the second integral on the righthand side of Eq. (D6) yields

$$
\begin{aligned}
& \int_{0}^{t-\bar{t}} \dot{X}\left(t-t^{\prime}\right) A\left(t^{\prime}\right) d t^{\prime} \\
& \quad \approx \sum_{k=1}^{i-\bar{l}}[X(i-k+1)-X(i-k)] d A(k) \\
& \quad \equiv X(i) d A(1)+I_{2}^{\prime},
\end{aligned}
$$

with moments

$$
d A(k) \equiv \frac{1}{2^{d} h} \int_{t_{k-1}}^{t_{k}} A\left(t^{\prime}\right) d t^{\prime},
$$

for $k=1, \ldots, i-\bar{\imath}$.

Finally, we approximate the time derivative $\dot{X}(t)$ in Eq. (D5) using a three-point backward difference formula [50],

$$
\dot{X}(i) \approx \frac{1}{2^{d} h}\left[\frac{1}{2} X(i-2)-2 X(i-1)+\frac{3}{2} X(i)\right] .
$$

Substitution of the approximations (D7), (D9), and (D11) into Eq. (D5) and solving the resulting equation for $X(i)$ yields

$$
X(i)=\frac{1}{\alpha}[\beta(i)-S], \quad i=n / 2+1, \ldots, n,
$$

where

$$
\begin{gathered}
\alpha \equiv \frac{3}{2} \frac{1}{2^{d} h}+d A(1), \\
\beta(i) \equiv \dot{B}(i)-\frac{1}{2^{d} h}\left[\frac{1}{2} X(i-2)-2 X(i-1)\right],
\end{gathered}
$$

and

$$
S \equiv A(i-\bar{\imath}) X(\bar{\imath})+I_{1}+I_{2}^{\prime} .
$$

Again, we consider $\bar{l}=\lfloor i / 2\rfloor$. The moments $d X(k)$ and $d A(k)$ are calculated and decimated as in Eqs. (C12), (C13), and (C14).

Equation (D12) provides an explicit approximated expression for the abstract functional relation (C4).

\section{APPENDIX E: TESTS OF THE NUMERICAL SOLUTION FOR THE PROBE CORRELATOR}

First we assess the quality of the numerical solution of Eq. (31) in the case of localized probes $\left(F<F_{c}\right)$. This is done by comparing the calculated values for $\Phi_{\mathbf{q}}^{\mathrm{s}}(t)$ at long times with the probe nonergodicity parameter $f_{\mathbf{q}}^{\mathrm{s}}$ provided by the fixed-point equation (54). The long-time limit $t \rightarrow \infty$ corresponds to $i=n, d=D$ in the decimated time grid (C1).

In the longitudinal direction, the quantities being compared are complex valued and we write $f_{q, \|}^{s}=f_{q, \|}^{\prime s}+i f_{q, \|}^{\prime \prime s}$ and $\Phi_{q, \|}^{s}(\infty)=\Phi_{q, \|}^{\prime s}(\infty)+i \Phi_{q, \|}^{\prime \prime s}(\infty)$. In the transverse direction $\Phi_{q, \perp}^{s, \|}(\infty)$ and $f_{q, \perp}^{s}$ are real valued.

In Fig. 13 we plot the relative errors

$$
\begin{aligned}
& e_{1}=\left|\Phi_{q, \|}^{\prime s}(\infty)-f_{q, \|}^{\prime s}\right| / f_{q, \|}^{\prime s}, \\
& e_{2}=\left|\Phi_{q, \|}^{\prime \prime s}(\infty)-f_{q, \|}^{\prime \prime s}\right| / f_{q, \|}^{\prime \prime s}, \\
& e_{3}=\left|\Phi_{q, \perp}^{s}(\infty)-f_{q, \perp}^{s}\right| / f_{q, \perp}^{s},
\end{aligned}
$$

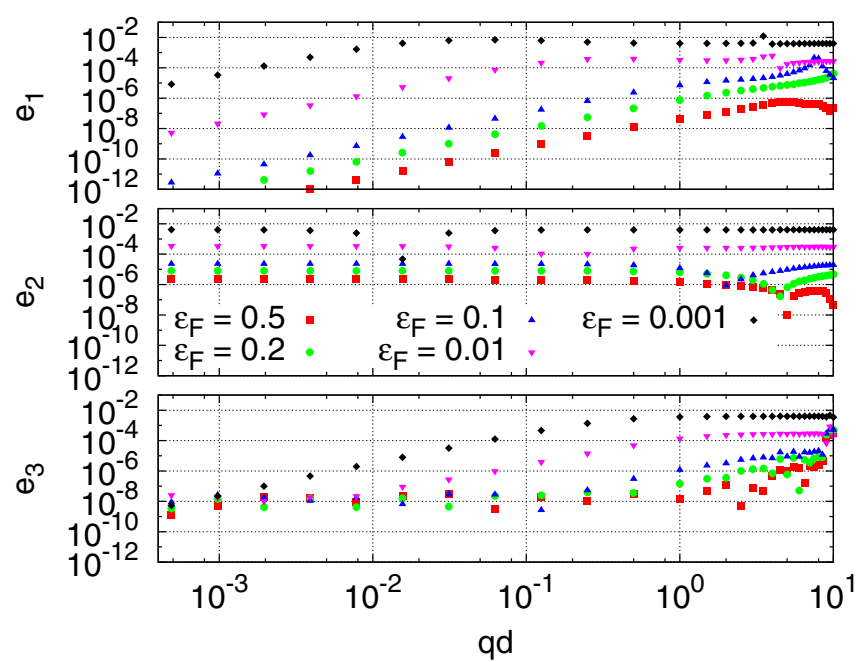

FIG. 13. Relative errors $e_{j}$ defined in Eq. (E1) as functions of $q d$ for different values of $\varepsilon_{\mathrm{F}}$ and host system at $\phi=0.537$.

as functions of the wave number $q$ for different values of relative distance $\varepsilon_{\mathrm{F}}=\left(F_{c}-F\right) / F_{c}$ from the critical force. Overall, the errors are small and increase as the critical force is approached. The largest observed errors (around 1\%) occur at $\varepsilon_{\mathrm{F}}=0.001$.

For delocalized probes $\left(F>F_{c}\right)$, we assess the accuracy of the numerical solution by comparing the quantities

$$
A_{\mathbf{q}}^{(0)}(\omega)=\frac{\tilde{\Phi}_{\mathbf{q}}^{\mathrm{s}}(\omega)}{1+i \omega \tilde{\Phi}_{\mathbf{q}}^{\mathrm{s}}(\omega)}, \quad A_{\mathbf{q}}^{(1)}(\omega)=\frac{1+\tilde{m}_{\mathbf{q}}(\omega)}{\Gamma_{\mathbf{q}}}
$$

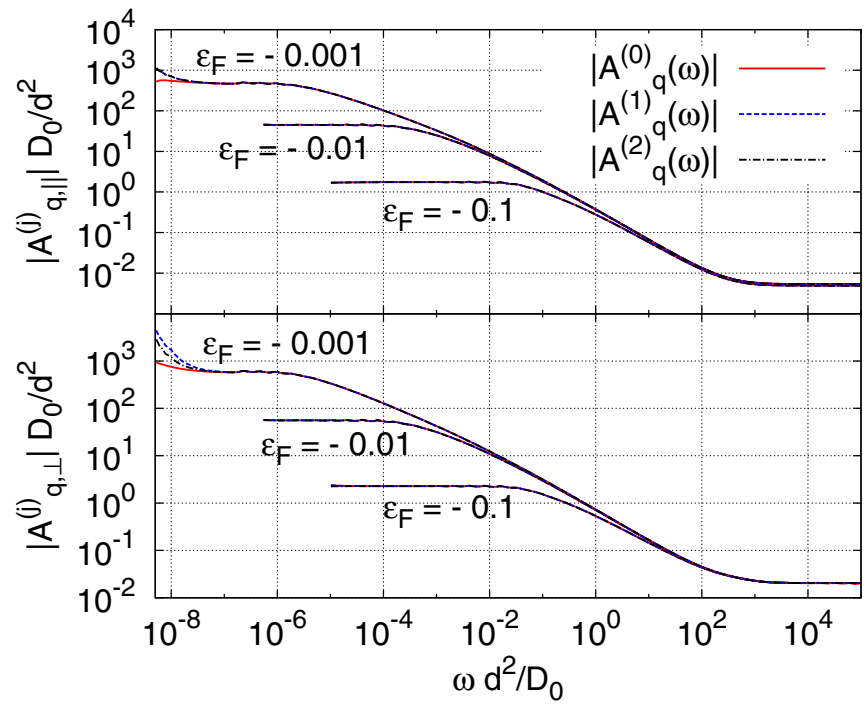

FIG. 14. Comparison of the quantities $\left|A_{\mathbf{q}}^{(j)}(\omega)\right|(j=0,1,2)$ defined in Eqs. (E2) and (E3) for different values of $\varepsilon_{\mathrm{F}}<0$ and for a wave vector close to the first peak of the host structure factor (at $\phi=0.516$ ), along the force direction (top), and perpendicular to it (bottom). The Fourier-Laplace transforms were evaluated numerically using Filon's quadrature formula [50]. 
and

$$
A_{\mathbf{q}}^{(2)}(\omega)=\frac{1}{\mathbf{L}_{\mathbf{q}}^{*} \cdot\left[\mathbf{1}+\tilde{\mathbf{m}}_{\mathbf{q}}(\omega)\right]^{-1} \cdot \mathbf{R}_{\mathbf{q}}},
$$

where $\tilde{\Phi}_{\mathbf{q}}^{\mathrm{s}}(\omega), \tilde{m}_{\mathbf{q}}(\omega)$, and $\tilde{\mathbf{m}}_{\mathbf{q}}(\omega)$ are the Fourier-Laplace transform [defined through Eq. (30) with $s=-i \omega$ ] of $\Phi_{\mathbf{q}}^{\mathrm{s}}(t)$, $m_{\mathbf{q}}(t)$, and $\mathbf{m}_{\mathbf{q}}(t)$, respectively. According to Eqs. (33) and (32) with $s=-i \omega, A_{\mathbf{q}}^{(0)}(\omega)=A_{\mathbf{q}}^{(1)}(\omega)=A_{\mathbf{q}}^{(2)}(\omega)$.

The quantities $\left|A_{\mathbf{q}}^{(j)}(\omega)\right|$ are compared in Fig. 14 for a wave vector $q$ around the first peak of the structure factor of the host (along and perpendicular to the force) and different values of the separation parameter $\varepsilon_{\mathrm{F}}$. The curves collapse in the full range of frequencies presented, up to the low-frequency plateau, which corresponds to the mean relaxation time of the correlators,

$$
\tau_{\mathbf{q}}=A_{\mathbf{q}}^{(j)}(\omega \rightarrow 0)=\int_{0}^{\infty} \Phi_{\mathbf{q}}^{\mathrm{s}}(t) d t
$$

The large-frequency limits $A_{\mathbf{q}}^{(j)}(\omega \rightarrow \infty)=1 / \Gamma_{\mathbf{q}}$ correspond to the short relaxation times.
[1] T. G. Mason and D. A. Weitz, Phys. Rev. Lett. 75, 2770 (1995)

[2] P. Cicuta and A. M. Donald, Soft Matter 3, 1449 (2007).

[3] L. Wilson and W. C. K. Poon, Phys. Chem. Chem. Phys. 13, 10617 (2011).

[4] A. M. Puertas and T. Voigtmann, J. Phys.: Condens. Matter 26, 243101 (2014).

[5] R. G. Larson, The Structure and Rheology of Complex Fluids (Oxford University Press, New York, 1999).

[6] A. M. Puertas and Th. Voigtmann, Complex Dynamics of GlassForming Liquids-A Mode-Coupling Theory (Oxford University Press, Oxford, UK, 2009).

[7] J. M. Brader, J. Phys.: Condens. Matter 22, 363101 (2010).

[8] M. Siebenbürger, M. Ballauff, and T. Voigtmann, Phys. Rev. Lett. 108, 255701 (2012).

[9] F. Varnik, L. Bocquet, and J.-L. Barrat, J. Chem. Phys. 120, 2788 (2004).

[10] A. Nicolas, K. Martens, L. Bocquet, and J.-L. Barrat, Soft Matter 10, 4648 (2014).

[11] J. L. Barrat, W. Götze, and A. Latz, J. Phys.: Condens. Matter 1, 7163 (1989).

[12] M. Fuchs, W. Götze, and M. R. Mayr, Phys. Rev. E 58, 3384 (1998).

[13] A. M. Puertas, J. Phys.: Condens. Matter 22, 104121 (2010).

[14] I. C. Carpen and J. F. Brady, J. Rheol. 49, 1483 (2005).

[15] T. M. Squires and J. F. Brady, Phys. Fluids 17, 073101 (2005).

[16] R. N. Zia and J. F. Brady, J. Fluid Mech. 658, 188 (2010).

[17] I. Gazuz, A. M. Puertas, T. Voigtmann, and M. Fuchs, Phys. Rev. Lett. 102, 248302 (2009).

[18] I. Gazuz and M. Fuchs, Phys. Rev. E 87, 032304 (2013).

[19] D. Winter, J. Horbach, P. Virnau, and K. Binder, Phys. Rev. Lett. 108, 028303 (2012).

[20] C. Harrer, Active and Nonlinear Microrheology of Dense Colloidal Suspensions, Ph.D. thesis, Universität Konstanz, 2013.

[21] C. J. Harrer, A. M. Puertas, and T. Voigtmann, Z. Phys. Chem. 226, 779 (2012).

[22] K. Chen, E. J. Saltzman, and K. S. Schweizer, Annu. Rev. Condens. Matter Phys. 1, 277 (2010).

[23] V. Kobelev and K. S. Schweizer, Phys. Rev. E 71, 021401 (2005).

[24] S. Lang, V. Boţan, M. Oettel, D. Hajnal, T. Franosch, and R. Schilling, Phys. Rev. Lett. 105, 125701 (2010).

[25] S. Lang, R. Schilling, and T. Franosch, J. Stat. Mech. (2013) P12007.

[26] D. Forster, Hydrodynamic Fluctuations, Broken Symmetry, and Correlation Functions, Advanced Book Classics (Perseus, New York, 1995).
[27] J. K. G. Dhont, An Introduction to Dynamics of Colloids (Elsevier, Amsterdam, 1996).

[28] B. Cichocki and W. Hess, Phys. A (Amsterdam, Neth.) 141, 475 (1987).

[29] K. Kawasaki, Phys. A (Amsterdam, Neth.) 215, 61 (1995).

[30] T. Franosch, M. Fuchs, W. Götze, M. R. Mayr, and A. P. Singh, Phys. Rev. E 56, 5659 (1997).

[31] M. Gruber, Microrheology Considering Parallel Relaxation Channels, Master's thesis, Universität Konstanz, 2014 .

[32] J.-P. Hansen and I. R. McDonald, Theory of Simple Liquids (Academic Press, San Diego, CA, 2006).

[33] T. Franosch, M. Fuchs, W. Götze, M. R. Mayr, and A. P. Singh, Phys. Rev. E 55, 7153 (1997).

[34] P. Habdas, D. Schaar, A. C. Levitt, and E. R. Weeks, Europhys. Lett. 67, 477 (2004).

[35] D. Andersson, D. Schaar, H. G. E. Hentschel, J. Hay, P. Habdas, and E. R. Weeks, J. Chem. Phys. 138, 12A520 (2013).

[36] C. Zhang, N. Gnan, T. G. Mason, E. Zaccarelli, and F. Scheffold, J. Stat. Mech. (2016) 094003.

[37] W. Paul and D. Y. Yoon, Phys. Rev. E 52, 2076 (1995).

[38] T. Voigtmann, A. M. Puertas, and M. Fuchs, Phys. Rev. E 70, 061506 (2004).

[39] F. Weysser, A. M. Puertas, M. Fuchs, and T. Voigtmann, Phys. Rev. E 82, 011504 (2010).

[40] K. N. Pham, A. M. Puertas, J. Bergenholtz, S. U. Egelhaaf, A. Moussaï, P. N. Pusey, A. B. Schofield, M. E. Cates, M. Fuchs, and W. C. K. Poon, Science 296, 104 (2002).

[41] D. Winter and J. Horbach, J. Chem. Phys. 138, 12 A512 (2013).

[42] P. Chaudhuri, L. Berthier, and W. Kob, Phys. Rev. Lett. 99, 060604 (2007).

[43] E. J. Saltzman and K. S. Schweizer, Phys. Rev. E 77, 051504 (2008).

[44] D. S. Fisher, Phys. Rev. B 31, 1396 (1985).

[45] A. M. Puertas, AIP Conf. Proc. 1319, 141 (2010).

[46] T. M. Squires and T. G. Mason, Annu. Rev. Fluid Mech. 42, 413 (2010).

[47] J. Zausch, J. Horbach, M. Laurati, S. U. Egelhaaf, J. M. Brader, T. Voigtmann, and M. Fuchs, J. Phys.: Condens. Matter 20, 404210 (2008).

[48] M. Fuchs, W. Götze, I. Hofacker, and A. Latz, J. Phys.: Condens. Matter 3, 5047 (1991).

[49] F. G. Tricomi, Integral Equations (Dover, Mineola, NY, 1985).

[50] M. Abramowitz and I. Stegun, Handbook of Mathematical Functions (Dover, Mineola, NY, 1965). 